Leila Hamdad • Ouafae Benrabah · Sophie Dabo-Niang

\title{
Exploring spectral density estimation for spatial linear process with mixing innovations
}

Received: 22 February 2018 / Accepted: 9 November 2018 / Published online: 27 November 2018 (C) The Author(s) 2018

\begin{abstract}
We study the asymptotic properties of the spectral density estimator (a periodogram) of a linear spatial process with alpha mixing innovations. A periodogram is a natural estimate of the spectral density. Under some conditions, a relation between the periodograms of innovations and that of the linear process is established in a spatial case. As the estimator of periodogram is inconsistent, a linear filter is introduced and convergence properties of the obtained smoother periodogram estimator are studied.
\end{abstract}

\section{Introduction}

Spatial statistics includes any statistical techniques which study phenomena observed on spatial sets. Such phenomena appear in a variety of fields, including soil science, geology, oceanography, econometrics, epidemiology, environmental science, forestry, image processing and many others.

Voluminous geographic data are being collected with modern acquisition techniques such as global positioning systems (GPS), high-resolution remote sensing, geographic information system (GIS), etc. Extracting unknown and unexpected information from these spatial data sets requires efficient methods that take into account the spatial dependencies [12]. Spatial data are characterized by their interdependence, which comes from the following assumption: The more objects are close to each other, the higher is the correlation between them [15]. Hence describing dependence is very important in spatial statistics. To do so, variogram and covariogram are usually used. In recent years, some authors developed spectral methods to describe this dependance [17] as spectral density. In time series, the periodogram is the non-parametric estimator of spectral density.

Spatial periodogram is a powerful tool for studying the properties of random fields observed on a lattice. It can be used in signal extraction from noisy random fields, in obtaining more sparse decomposition of digital image requiring less storage space, in detecting hidden periodicities, prediction or smoothing and so on. Spatial spectral methods have been applied to astronomy (see [1]) and meteorology [4] among others.

The time series have been developed in the spatial context because of their interest in environmental science, medical research, ecology among others. Some authors have been interested in developing methods and theory

L. Hamdad $(\varangle)$

Laboratoire LCSI, École Nationale Supérieure d'Informatique (ESI), BP68, Oued El Smar, El Harrach, Algiers, Algeria

E-mail:1_hamdad@esi.dz

O. Benrabah

Laboratoire LMPA, Université de Littoral, Côte d'Opale, Dunkerque, France

E-mail: Ouafae.Benrabah@univ-littoral.fr

S. Dabo-Niang

Laboratoire LEM CNRS 9221, University of Lille, INRIA-Lille, MODAL TEAM, Tourcoing, France

E-mail: sophie.dabo@univ-lille.fr 
to deal with this type of problem, see for examples [2,3,8,13], and most recently, [11]. A discussion on several models is done to deal with analysis of spatial data [16].

In this work, we are interested by the properties of spatial linear processes with alpha mixing innovations and mainly on periodogram. The lack of spatial asymptotic results of the periodogram of spectral density of these processes motivates this work. Hence, we study the asymptotic properties of this periodogram. A periodogram is a natural estimate of the spectral density. Under some conditions, a relation between the periodogram of innovations and the periodogram of linear process is established in a spatial case. As the estimator of periodogram is inconsistent, a linear filter is introduced and convergence properties of the obtained smoother periodogram estimator are studied.

The periodogram in the non-spatial case $(N=1)$ has been studied by [6], when the innovations are independent while [5] has studied the case where innovations are strongly mixing. We extend this last work to random fields $(N>1)$. Some recent papers deal with spatial periodogram smoothing but there is no work concerning weak processes. Different filters are used in literature, see, for example, [19] which focuses on kernel estimator of spectral density, with optimal smoothing number estimated from the data. The author studied consistency and asymptotic distribution of this estimator, with an automatic estimate of this smoothing number. In [9], the authors have studied asymptotic properties of smoothed non-parametric kernel spectral density estimator in the case of continuous stationary spatial process under shrinking asymptotic framework. This process is mixing but not necessarily derived from weak process as in the present work. They considered the bias and variances terms for tapered and untapered estimators, and obtained optimal bandwidth which minimizes the average mean squared error.

Let $\mathbb{Z}^{N}, N \geq 1$, denote the integer lattice points in the $N$-dimensional Euclidean space. Let the process $\left(X_{\mathbf{t}}, \mathbf{t} \in \mathbb{Z}^{N}\right)$ be a stationary spatial linear process defined on a probability space $(\Omega, \mathcal{A}, P)$, of unknown spectral density $f_{X}: X_{\mathbf{t}}=\sum_{\mathbf{s} \in \mathbb{Z}^{N}} a_{\mathbf{s}} Z_{\mathbf{t}-\mathbf{s}}, \mathbf{t} \in \mathbb{Z}^{N}$, where the $\left(Z_{\mathbf{t}}\right)$ is a strictly stationary process with unknown spectral density $f_{Z}$. $Z_{\mathbf{t}}$ are real-valued $\alpha$ mixing, and identically distributed and uncorrelated random variables with zero mean and finite second moment $\sigma^{2}$. The spectral density of $\left(X_{\mathbf{t}}\right)$ is given by

$$
f_{X}(\omega)=\frac{1}{(2 \pi)^{N}} \sum_{\mathbf{k} \in \mathbb{Z}^{N}} \gamma(\mathbf{k}) \exp \left(-i \omega^{T} \mathbf{k}\right), \omega \in[-\pi, \pi]^{N},
$$

where $T$ is the transpose operator and $\gamma(\mathbf{k})=\operatorname{Cov}\left(X_{\mathbf{k}+\mathbf{u}}, X_{\mathbf{u}}\right)$ is the auto-covariance of $\left(X_{\mathbf{t}}, \mathbf{t} \in \mathbb{Z}^{N}\right)$.

A point $\mathbf{k}$ in $\mathbb{Z}^{N}$ will be referred to as a site and written as $\mathbf{k}=\left\langle k_{1}, \ldots, k_{N}\right\rangle$. Let $\mathbf{n}=\left\langle n_{1}, \ldots, n_{N}\right\rangle$, we write $\mathbf{n} \rightarrow \infty$ if $\min \left\{n_{k}\right\} \rightarrow \infty$ and $1<j, k<N$. Define $\widehat{\mathbf{n}}=n_{1} \cdots n_{N}$ as sample size. All limits are taken as $\mathbf{n} \rightarrow \infty$. $\left(X_{\mathbf{t}}, \mathbf{t} \in \mathbb{Z}^{N}\right)$ is observed over the rectangular domain $I_{\mathbf{n}}=$ $\left\{\mathbf{i}=\left\{i_{1}, \ldots, i_{N}\right\} \in \mathbb{Z}^{N} ; 1 \leq i_{k} \leq n_{k}, k=1, \ldots, N\right\}$. In what follows, let $\mathbf{i} \leq \mathbf{j}$ denote $\exists k, i_{k} \leq j_{k}, i_{l}=$ $j_{l}, l=1, \ldots, k-1$ (the lexicographic order). A simple natural estimator of $f_{X}\left(\omega_{\mathbf{j}}\right)$ appears to be $\frac{I_{\mathbf{n}, X}\left(\omega_{\mathbf{j}}\right)}{(2 \pi)^{N}}$, where $I_{\mathbf{n}, X}\left(\omega_{\mathbf{j}}\right)$ is the spatial periodogram of $\left(X_{\mathbf{t}}, \mathbf{t} \in \mathbb{Z}^{N}\right)$ defined at frequency $\omega_{\mathbf{j}}=\left(\omega_{j_{1}}=\frac{2 \pi j_{1}}{n_{1}}, \ldots, \omega_{j_{N}}=\right.$ $\left.\frac{2 \pi j_{N}}{n_{N}}\right), \omega_{\mathbf{j}} \in[-\pi, \pi]^{N}$ by

$$
I_{\mathbf{n}, X}\left(\omega_{\mathbf{j}}\right)=\frac{1}{\widehat{\mathbf{n}}}\left|\sum_{\mathbf{t} \in I_{\mathbf{n}}} X_{\mathbf{t}} e^{-i \omega_{\mathbf{j}}^{T} \mathbf{t}}\right|^{2}
$$

If $\gamma($.$) is an absolutely summable auto-covariance function of the process \left(X_{\mathbf{t}}, \mathbf{t} \in \mathbb{Z}^{N}\right)$, then $f_{X}$ is continuous and the definition (2) is equivalent to

$$
I_{\mathbf{n}, X}\left(\omega_{\mathbf{j}}\right)=\sum_{\mathbf{k} \in S} \widehat{\gamma}(\mathbf{k}) \exp \left(-i \omega_{\mathbf{j}}^{T} \mathbf{k}\right), \omega \in[-\pi, \pi]^{N},
$$

where $S=\left\{\mathbf{k} ; k_{j}=1-n_{j}, \ldots, n_{j}-1, j=1, \ldots, N\right\}$ and

$$
\widehat{\gamma}(\mathbf{k})=\frac{1}{\widehat{\mathbf{n}}} \sum_{\mathbf{t} \in I(\mathbf{k})}\left(X_{\mathbf{t}}-\bar{X}\right)\left(X_{\mathbf{t}+\mathbf{k}}-\bar{X}\right),
$$


with mean $\bar{X}=\frac{1}{\hat{n}} \sum_{\mathbf{t} \in I_{\mathbf{n}}} X_{\mathbf{t}}$ and where $I(\mathbf{k})=\left\{\mathbf{t} \in I_{\mathbf{n}}, \mathbf{t}+\mathbf{k} \in I_{\mathbf{n}}\right\}$. Hence the natural spectral density estimator is given by

$$
\widehat{f}_{X}(\omega)=\frac{1}{(2 \pi)^{N}} \sum_{\mathbf{k} \in S} \widehat{\gamma}(\mathbf{k}) \exp \left(-i \omega^{T} \mathbf{k}\right), \omega \in[-\pi, \pi]^{N} .
$$

As in the non-spatial case $(N=1)$, this estimator is unbiased but not consistent. The consistent estimator is obtained by applying a linear filter to smooth the periodogram.

In this paper, we will deal with asymptotic properties of spectral density estimates $\widehat{f}_{X}$ of $\left(X_{\mathbf{t}}, \mathbf{t} \in \mathbb{Z}^{N}\right)$ using observations on $I_{\mathbf{n}}$ and the following conditions:

Mixing conditions: Consider the $\alpha$-mixing coefficient of the field $\left(Z_{\mathbf{t}}, \mathbf{t} \in \mathbb{Z}^{N}\right)$. The spatial dependence of the process will be measured by this $\alpha$ mixing defined by

$$
\alpha(v)=\sup _{\mathbf{u}, \mathbf{u}^{\prime} \in Z^{N},\left\|\mathbf{u}-\mathbf{u}^{\prime}\right\|=v} \alpha\left(\sigma\left(Z_{\mathbf{u}}\right), \sigma\left(Z_{\mathbf{u}}^{\prime}\right)\right), v \geq 0,
$$

where, for $\mathcal{B}$ and $\mathcal{C}$, two $\sigma$-fields of $\mathcal{A}: \alpha(\mathcal{B}, \mathcal{C})=\sup _{B \in \mathcal{B}, C \in \mathcal{C}}|P(B \cap C)-P(B) P(C)|$. In the following, we suppose that the process is geometrically strongly mixing (GSM), that is, there exist $\beta>0$ and a constant $C>0$ such that

$$
\alpha(u) \leq C u^{-\beta}, u>0 .
$$

This paper is organized as follows. In Sect. 2, we deal with asymptotic properties of the spatial periodograms of $\left(Z_{\mathbf{t}}\right)$ and $\left(X_{\mathbf{t}}\right)$. In Theorem 1 , we first give a result that concerns an asymptotic distribution of the periodogram of innovations. Then in Theorem 2, we establish a relation between the periodogram of the innovations and that of $\left(X_{\mathbf{t}}\right)$; hence, the convergence of the periodogram of $\left(X_{\mathbf{t}}\right)$ to exponential independent variables is given in Theorem 3. In Sect. 3, we study the convergence of a smoothed periodogram of $\left(X_{\mathbf{t}}\right)$. Finally, Sect. 4 is devoted to some numerical results of the proposed periodogram.

\section{Convergence of the periodogram}

To estimate $f_{X}$ at arbitrary non-zero frequencies in the interval $[-\pi, \pi]^{N}$, we need to extend the domain of $I_{\mathbf{n}, X}$ to the whole interval $[-\pi, \pi]^{N}$. The periodogram is then defined as a piecewise constant function which coincides with (2) in frequency $\omega_{\mathbf{j}}$. For any $\omega \in[-\pi, \pi]^{N}$, the periodogram is defined as follows (using (2)):

$$
I_{\mathbf{n}, X}(\omega)=\left\{\begin{array}{l}
I_{\mathbf{n}, X}\left(\omega_{\mathbf{j}}\right), \text { if } \omega_{\mathbf{j}_{k}}-\frac{\pi}{n_{k}}<\omega_{k}<\omega_{\mathbf{j}_{k}}+\frac{\pi}{n_{k}} \text { and } \omega \in[0, \pi]^{N}, 1 \leq k \leq N \\
I_{\mathbf{n}, X}(-\omega), \text { if } \omega \in[-\pi, 0]^{N}
\end{array}\right.
$$

Let $g(\mathbf{n}, \omega)=\left(g\left(n_{1}, \omega\right), \ldots, g\left(n_{N}, \omega\right)\right)$ be the multiple of $\left(\frac{2 \pi}{n_{1}}, \ldots, \frac{2 \pi}{n_{N}}\right)$ closest to $\omega$. And let $g(\mathbf{n}, \omega)=$ $g(\mathbf{n},-\omega)$ for $\omega \in[-\pi, 0]^{N}$. Then $I_{\mathbf{n}, X}(\omega)=I_{\mathbf{n}, X}(g(\mathbf{n}, \omega))$. Set, for $w_{\mathbf{j}} \in[-\pi, \pi]^{N}$

$$
\begin{aligned}
& A\left(\omega_{\mathbf{j}}\right)=\left(\frac{2}{\widehat{\mathbf{n}}}\right)^{\frac{1}{2}} \sum_{\mathbf{t} \in I_{\mathbf{n}}} X_{\mathbf{t}} \cos \omega_{\mathbf{j}}^{T} \mathbf{t}, \\
& B\left(\omega_{\mathbf{j}}\right)=\left(\frac{2}{\widehat{\mathbf{n}}}\right)^{\frac{1}{2}} \sum_{\mathbf{t} \in I_{\mathbf{n}}} X_{\mathbf{t}} \sin \omega_{\mathbf{j}}^{T} \mathbf{t} .
\end{aligned}
$$

Then $I_{\mathbf{n}, X}\left(\omega_{\mathbf{j}}\right)=\frac{A^{2}\left(\omega_{\mathbf{j}}\right)+B^{2}\left(\omega_{\mathbf{j}}\right)}{2}$. Let $\lambda_{1} \leq \cdots \leq \lambda_{m} \in[0, \pi]^{N}$ be the ordered sequence of $\omega_{\mathbf{k}^{1}}, \ldots, \omega_{\mathbf{k}^{m}}$, $m \geq 1$ with $\omega_{\mathbf{k}^{j}}=\left(\frac{2 \pi k_{1}^{j}}{n_{1}}, \ldots, \frac{2 \pi k_{N}^{j}}{n_{N}}\right), \forall j=1, \ldots, m, \mathbf{k}^{j}=\left(k_{1}^{j}, \ldots, k_{N}^{j}\right)$. Then, $\exists \mathbf{k}^{l}$ such that $\lambda_{\mathbf{j}}=\left(\lambda_{\mathbf{j}, 1}, \ldots, \lambda_{\mathbf{j}, N}\right)=\omega_{\mathbf{k}^{l}}$.

To establish the Theorems 1 and 2 below, we need the following assumptions: 
Assumption 1 For $\gamma>\frac{4 \rho-4}{\rho}$ and all $2 \leq k \leq \rho$ such that $\rho>2, M_{2 \gamma}=\max _{\mathbf{t}}\left(E\left[\left|Z_{\mathbf{t}}\right|^{2 \gamma}\right]\right)^{\frac{1}{2 \gamma}}<\infty$ and $E\left[\left|Z_{\mathbf{t}}\right|^{k}\right] \leq C$ uniformly in where $\mathbf{t}$ and $0<C<\infty$ are a constant.

Assumption 2 Assume that the mixing coefficient of $\left(Z_{\mathbf{t}}\right)$ satisfies

$\lim _{n \rightarrow \infty} n^{\kappa} \sum_{t=n}^{\infty} t^{N \frac{\rho}{2}-1} \alpha(t)^{\frac{\gamma \rho-4}{\gamma \rho}}=0$, for some constant $\kappa>\frac{2 N \rho(\gamma \rho-4)}{8+2 \rho(\gamma-4)}$.

These two assumptions hold in such problems and under our conditions on mixing process (geometric). They are used to manipulate our spatial inequalities as in Gao [10].

Let $I_{\mathbf{n}, Z}$ be the periodogram of $\left(Z_{\mathbf{t}}\right)$ obtained by replacing $\left(X_{\mathbf{t}}\right)$ by $\left(Z_{\mathbf{t}}\right)$ in (6). We begin by a consistency result of $I_{\mathbf{n}, Z}$ for all $t$.

Theorem 1 Assume that for $\gamma>2$ and $m_{\gamma}>0, E\left(\left|Z_{\mathbf{t}}\right|^{2 \gamma}\right)=m_{\gamma}, \mathbf{t} \in \mathbb{Z}^{N}$ and $\sum_{i} i^{N-1} \alpha(i)^{\frac{\gamma-2}{\gamma}}<\infty$.

(i) Under Assumptions 1 and 2 , and if $\alpha(k) \simeq k^{-\beta}$ for some $\beta>\frac{\gamma N}{\gamma-2}$, then for $\lambda_{1}, \ldots, \lambda_{m} \in[0, \pi]^{N}$, the random vector $\left(I_{\mathbf{n}, Z}\left(\lambda_{1}\right), \ldots, I_{\mathbf{n}, Z}\left(\lambda_{m}\right)\right)$ converges as $\mathbf{n} \rightarrow \infty$ to a vector of exponentially independent distributed random variables, each with mean $\sigma^{2}$.

(ii) If $E Z_{\mathbf{1}}^{4}=\theta \sigma^{4}<\infty$, then $\operatorname{Cov}\left(I_{\mathbf{n}, Z}\left(\lambda_{\mathbf{j}}\right), I_{\mathbf{n}, Z}\left(\lambda_{\mathbf{l}}\right)\right)$

$$
=\left\{\begin{array}{l}
\widehat{\mathbf{n}}^{-1}(\theta-3) \sigma^{4}+2 \sigma^{4}+O\left(\widehat{\mathbf{n}}^{-1}\right) \text { if } \lambda_{\mathbf{j}}=\lambda_{\mathbf{l}} \text { and }, \lambda_{\mathbf{j}, k}=0 \text { or } \pi, \forall k=1, \ldots, N, \\
\widehat{\mathbf{n}}^{-1}(\theta-3) \sigma^{4}+\sigma^{4}+O\left(\widehat{\mathbf{n}}^{-1}\right) \text { if } \lambda_{\mathbf{j}}=\lambda_{\mathbf{l}} \text { and } 0<\lambda_{\mathbf{j}, k}<\pi, \forall k=1, \ldots, N, \\
\left.\widehat{n}^{4}+\widehat{\mathbf{n}}^{-1}\right) \text { if } \lambda_{\mathbf{j}} \neq \lambda_{\mathbf{I}} .
\end{array}\right.
$$

Proof For an arbitrary $\lambda \in[0, \pi]^{N}$, define $A(\lambda)=A(g(\mathbf{n}, \lambda))$ and $B(\lambda)=B(g(\mathbf{n}, \lambda))$, with $Z_{\mathbf{t}}$ replacing $X_{\mathbf{t}} ;$ since

$$
I_{Z}\left(\lambda_{\mathbf{j}}\right)=\frac{A^{2}\left(\lambda_{\mathbf{j}}\right)+B^{2}\left(\lambda_{\mathbf{j}}\right)}{2},
$$

it suffices to show that

$$
\left(A\left(\lambda_{1}\right), B\left(\lambda_{1}\right), \ldots, A\left(\lambda_{m}\right), B\left(\lambda_{m}\right)\right)
$$

converges in distribution to a centered Gaussian random vector with covariance matrix $\sigma^{2} I_{2 m} . I_{2 m}$ is the $2 m \times 2 m$ identity matrix.

Let $u_{1}, \ldots, u_{m}$ and $v_{1}, \ldots, v_{m} \in \mathbb{R}$, be fixed. Then set the random variables

$$
Y_{\mathbf{t}, \mathbf{n}}=\sum_{i=1}^{m} \sqrt{2}\left(u_{i} \cos \lambda_{i}^{T} \mathbf{t}+v_{i} \sin \lambda_{i}^{T} \mathbf{t}\right) Z_{\mathbf{t}}
$$

and

$$
S_{\mathbf{n}}=\sum_{\mathbf{t} \in \mathbb{Z}^{N}} Y_{\mathbf{t}, \mathbf{n}}
$$

To use the Cramer-Wold Theorem, we need the linear combination of the coordinates of (8). Assume for some integers $r_{1}, \ldots, r_{N}$, we have $n_{1}=r_{1}(p+q), \ldots, n_{N}=r_{N}(p+q), q<p$. The method of proof consists to define following large and small blocks used in [18]. Let,

$$
\begin{aligned}
U(1, \mathbf{n}, x, \mathbf{j}) & =\sum_{\substack{t_{k}=j_{k}(p+q)+1 \\
k=1, \ldots, N \\
j_{k}(p+q)+p}}^{j_{k}(p+q)+p} Y_{\mathbf{t}, \mathbf{n}}(x), \\
U(2, \mathbf{n}, x, \mathbf{j}) & =\sum_{\substack{t_{k}=j_{k}(p+q)+1 \\
k=1, \ldots, N-1}}^{\left(j_{N}+1\right)(p+q)} \sum_{t_{N}=j_{N}(p+q)+p+1} Y_{\mathbf{t}, \mathbf{n}}(x),
\end{aligned}
$$




$$
\begin{aligned}
& U(3, \mathbf{n}, x, \mathbf{j})=\sum_{\substack{t_{k}=j_{k}(p+q)+1 \\
k=1, \ldots, N-2}}^{j_{k}(p+q)+p} \sum_{t_{N-1}=j_{N-1}(p+q)+p+1}^{\left(j_{N-1}+1\right)(p+q)} \sum_{t_{N}=j_{N}(p+q)+1}^{j_{N}(p+q)+p} Y_{\mathbf{t}, \mathbf{n}}(x), \\
& U(4, \mathbf{n}, x, \mathbf{j})=\sum_{\substack{j_{k}(p+q)+p \\
t_{k}=j_{k}(p+q)+1 \\
k=1, \ldots, N-2}}^{\left.j_{t_{N-1}}=j_{N-1}(p+q)+p+1\right)(p+q)} \sum_{t_{N}=j_{N}(p+q)+p+1}^{\left(j_{N}+1\right)(p+q)} Y_{\mathbf{t}, \mathbf{n}}(x),
\end{aligned}
$$

And so on. Note that

$$
U\left(2^{N-1}, \mathbf{n}, x, \mathbf{j}\right)=\sum_{\substack{t_{k}=j_{k}(p+q)+p+1 \\ k=1, \ldots, N-1}}^{\left(j_{k}+1\right)(p+q)} \sum_{t_{N}=j_{N}(p+q)+1}^{j_{N}(p+q)+p} Y_{\mathbf{t}, \mathbf{n}}(x) .
$$

Finally,

$$
U\left(2^{N}, \mathbf{n}, x, \mathbf{j}\right)=\sum_{\substack{t_{k}=j_{k}(p+q)+p+1 \\ k=1, \ldots, N}}^{\left(j_{k}+1\right)(p+q)} Y_{\mathbf{t}, \mathbf{n}}(x) .
$$

Define

$$
T(\mathbf{n}, x, i)=\sum_{\substack{j_{k}=0 \\ k=1, \ldots, N}}^{r_{k-1}} U(i, \mathbf{n}, x, \mathbf{j}), \quad \text { for } \quad 1 \leq i \leq 2^{N} .
$$

Clearly,

$$
S_{\mathbf{n}}=\sum_{i=1}^{2^{N}} T(\mathbf{n}, x, i)
$$

$T(\mathbf{n}, x, 1)$ is the sum of random variables in large blocks and $T(\mathbf{n}, x, i)$ for $2 \leq i \leq 2^{N}$ the sum for random variables in small blocks. If it is not the case that $n_{1}=r_{1}(p+q), \ldots, n_{N}=r_{N}(p+q)$ for some integers $r_{1}, \ldots, r_{N}$, the term $T\left(\mathbf{n}, x, 2^{N}+1\right)$ can be added. This term will not change the proof much (see [18]).

Consider $T(\mathbf{n}, x, 1)$, we enumerate in the arbitrary way the $r=r_{1} \times \cdots \times r_{N}$ terms $U(1, \mathbf{n}, x, j)$ of sum of $T(\mathbf{n}, x, 1)$ which we denote $W_{1}, \ldots, W_{r}$. Note that the $U(1, \mathbf{n}, x, j)$ is measurable with respect to the $\sigma$-field generated by $Y_{\mathbf{t}}$, with $\mathbf{t}$ such that $j_{k}(p+q)+1 \leq t_{k} \leq j_{k}(p+q)+p$. Then distinct sets of sites $I(1, \mathbf{n}, x, j)=\left\{\mathbf{t} / j_{k}(p+q)+1 \leq t_{k} \leq j_{k}(p+q)+p\right\}$ are far apart by distance of at least $q$ and it contains $p^{N}$ sites. And note that $r=\widehat{\mathbf{n}}(p+\bar{q})^{-N} \leq \widehat{\mathbf{n}} p^{-N}$.

We have

$$
\begin{aligned}
\left\|Y_{\mathbf{t}, \mathbf{n}}\right\|_{2 \gamma}^{2 \gamma} & =E\left(\sum_{i=1}^{m} \sqrt{2}\left(u_{i} \cos \omega^{T} \mathbf{t}+v_{i} \sin \omega^{T} \mathbf{t}\right) Z_{\mathbf{t}}\right)^{2 \gamma} \\
& \leq 2^{\gamma} E Z_{\mathbf{t}}^{2 \gamma}\left(\sum_{i=1}^{m}\left(\left|u_{i}\right|+\left|v_{i}\right|\right)\right)^{2 \gamma}=b
\end{aligned}
$$

such that $b$ is a positive constant. Then

$$
\left\|W_{m}\right\|_{2 \gamma} \leq b p^{N}, \quad \text { for all } m=1, \ldots, r .
$$

According to Lemma 4.4 in [7], there exist independent random variables $W_{1}^{*}, \ldots, W_{r}^{*}$ such that for all $m=1, \ldots, r$

$$
E\left|W_{m}-W_{m}^{*}\right| \leq 2 b p^{N} \alpha(q) .
$$


We have

$$
\left.P\left(\left|\sum_{i=1}^{r} W_{i}\right|>\epsilon\right) \leq P\left(\left|\sum_{i=1}^{r}\left(W_{m}-W_{m}^{*}\right)\right|>\frac{\epsilon}{2}\right)+P\left(\mid \sum_{i=1}^{r} W_{m}^{*}\right) \mid>\frac{\epsilon}{2}\right) .
$$

Therefore, according to Markov inequality, we have

$$
P\left(\left|\sum_{i=1}^{r}\left(W_{m}-W_{m}^{*}\right)\right|>\frac{\epsilon}{2}\right) \leq 2 \sum_{i=1}^{r} \frac{E\left|W_{m}-W_{m}^{*}\right|}{\epsilon} .
$$

Hence from Eq. (12),

$$
P\left(\left|\sum_{i=1}^{r}\left(W_{m}-W_{m}^{*}\right)\right|>\frac{\epsilon}{2}\right) \leq \frac{2 r P^{N} \alpha(q)}{\epsilon} .
$$

Set $q=o\left(\widehat{n}^{c}\right)$, such that $c \in[0,1]$. Then $\alpha(q)=\widehat{n}^{-\beta c}$ where $\beta>\frac{\gamma N}{\gamma-2}$.

Hence, we have

$$
P\left(\left|\sum_{i=1}^{r}\left(W_{m}-W_{m}^{*}\right)\right|>\frac{\epsilon}{2}\right) \leq \frac{2 \widehat{n}^{-\beta c}}{\widehat{n}^{\epsilon}}=\frac{\widehat{n}^{1-\beta c}}{\epsilon}
$$

If we consider now

$$
\Delta_{N}=\frac{\sum_{m=1}^{r} W_{m}^{*}}{\sqrt{r p^{N}}}-\frac{\sum_{m=1}^{r} W_{m}}{\sqrt{r p^{N}}} .
$$

It follows that

$$
\begin{aligned}
P\left(\left|\Delta_{N}\right|>\epsilon\right) & \leq \sum_{m=1}^{r} P\left(\left|W_{m}-W_{m}^{*}\right|>\epsilon \sqrt{\frac{p^{N}}{r}}\right) \\
& \leq \frac{r^{\frac{1}{2}} p^{\frac{-N}{2}} \widehat{n}^{1-\beta c}}{\epsilon} \\
& \leq \frac{r^{\frac{3}{2}-\beta c} p^{\frac{N}{2}-\beta N c}}{\epsilon} \\
& =O\left(p^{N^{\frac{\gamma}{4 \gamma+1}}} r^{\frac{5 \gamma+1}{4 \gamma+1}} \alpha(q)^{\frac{4 \gamma}{4 \gamma+1}}\right) .
\end{aligned}
$$

Now, we are going to prove the asymptotic normality of $\left(r p^{N}\right)^{\frac{-1}{2}} \sum W_{m}^{*}$. First, we verify following Lyapounouv conditions. That is, for some $\rho>2$, we verify that

$$
\Pi=\frac{\sum_{m=1}^{r} E\left(\left|W_{m}^{*}\right|^{\rho}\right)}{\left(r \operatorname{Var}\left(W_{m}^{*}\right)\right)^{\frac{\rho}{2}}} \rightarrow 0
$$

We have

$$
\operatorname{Var}\left(W_{m}^{*}\right)=\operatorname{Var}\left(\sum_{\substack{t_{k}=j_{k}(p+q)+1 \\ k=1, \ldots, N}}^{j_{k}(p+q)+p} Y_{\mathbf{t}, \mathbf{n}}(x)\right)
$$




$$
=\operatorname{Var}\left(\sum_{\substack{t_{k}=1 \\ k=1, \ldots, N}}^{p} \sum_{i=1}^{m} \sqrt{2}\left(u_{i} \cos \lambda^{T} \mathbf{t}+v_{i} \sin \lambda^{T} \mathbf{t}\right) Z_{\mathbf{t}}\right) .
$$

Set $A_{\mathbf{t}}=\sum_{i=1}^{m} \sqrt{2}\left(u_{i} \cos \lambda^{T} \mathbf{t}+v_{i} \sin \lambda^{T} \mathbf{t}\right)$ and $B_{\mathbf{j}}=\sum_{i=1}^{m} \sqrt{2}\left(u_{i} \cos \lambda^{T} \mathbf{j}+v_{i} \sin \lambda^{T} \mathbf{j}\right)$.

We recall that $\left|\sum_{i=1}^{m} \sqrt{2}\left(u_{i} \cos \omega^{T} \mathbf{t}+v_{i} \sin \omega^{T} \mathbf{t}\right)\right|<\left|\sum_{i=1}^{m}\right| u_{i}|+| v_{i}||=C$. Then

$$
\begin{aligned}
\operatorname{Var}\left(W_{m}^{*}\right) & =\operatorname{Var} Z_{1} \sum_{\substack{t_{k}=1 \\
k=1, \ldots, N}}^{p}\left(\sum_{i=1}^{m} \sqrt{2}\left(u_{i} \cos \lambda^{T} \mathbf{t}+v_{i} \sin \lambda^{T} \mathbf{t}\right)\right)^{2}+\sum_{\substack{t_{k}=1 \\
k=1, \ldots, N}}^{p} \sum_{\substack{j_{k}=1 \\
k=1, \ldots, N}}^{p} A_{\mathbf{t}} B_{\mathbf{j}} \operatorname{Cov}\left(Z_{\mathbf{t}}, Z_{\mathbf{j}}\right) \\
& \leq 2 \sigma^{2} \sum_{\substack{t_{k}=1 \\
k=1, \ldots, N}}^{p}\left(\sum_{i=1}^{m} \sqrt{2}\left(u_{i} \cos \lambda^{T} \mathbf{t}+v_{i} \sin \lambda^{T} \mathbf{t}\right)\right)^{2}+C \sum_{\substack{t_{k}=1 \\
k=1, \ldots, N}}^{p} \sum_{\substack{j_{k}=1 \\
k=1, \ldots, N \\
t_{k} \neq j_{k}}}^{p}\left|\operatorname{Cov}\left(Z_{\mathbf{t}}, Z_{\mathbf{j}}\right)\right| \\
& \leq 2 C \sigma^{2} p^{N}+C \sum_{\substack{t_{k}=1 \\
k=1, \ldots, N}}^{p} \sum_{\substack{j_{k}=1 \\
k=1, \ldots, N \\
t_{k} \neq j_{k}}}^{p} \alpha(\|\mathbf{t}-\mathbf{j}\|)^{\frac{\gamma-2}{\gamma}} \\
& \leq 2 C \sigma^{2} p^{N}+C p^{N} \sum_{\substack{i_{k}=1 \\
k=1, \ldots, N}}^{p} \alpha(\|\mathbf{i}\|)^{\frac{\gamma-2}{\gamma}} .
\end{aligned}
$$

Hence

$$
r \operatorname{Var}\left(W_{m}^{*}\right) \leq 2 C \sigma^{2} r p^{N}+C r p^{N} \sum_{i=1}^{p} i^{N-1} \alpha(i)^{\frac{\gamma-2}{\gamma}} .
$$

Supposing that $\sum_{i=1}^{\infty} i^{N-1} \alpha(i)^{\frac{\gamma-2}{\gamma}}<\infty$, then

$$
r \operatorname{Var}\left(W_{m}^{*}\right) \sim 2 C \sigma^{2} r p^{N} .
$$

We will now focus our attention on the numerator of $\Pi$, that is, $\sum E\left(\left|W_{m}^{*}\right|^{\rho}\right)$. We have by Theorem 2.1 of [10] with $0<D<p^{N}$ and under Assumptions 1 and 2,

$$
\begin{aligned}
E\left(\left|W_{m}^{*}\right|^{\rho}\right)=E\left|\sum_{\substack{t_{k}=j_{k}(p+q)+1 \\
k=1, \ldots, N}}^{j_{k}(p+q)+p} Y_{\mathbf{t}, \mathbf{n}}(x)\right|^{\rho} & \leq p^{N} M_{2 \gamma}^{2} \sum_{t=D}^{\infty} t^{N \frac{\rho}{2}-1} \alpha(t)^{\frac{\gamma-2}{\gamma}}+\sum_{j=1}^{\frac{\rho}{2}} p^{N j} D^{\rho-1} C_{\theta}^{j} \\
& \leq p^{N} D^{-\kappa} D^{\kappa} M_{2 \gamma}^{2} \sum_{t=D}^{\infty} t^{N \frac{\rho}{2}-1} \alpha(t)^{\frac{\gamma-2}{\gamma}}+\sum p^{N j} D^{\rho-1} C_{\theta}^{j} \\
& \leq p^{N} D^{-\kappa} D^{\kappa} M_{2 \gamma}^{2} \sum_{t=D}^{\infty} t^{N \frac{\rho}{2}-1} \alpha(t)^{\frac{\gamma-2}{\gamma}}+D^{\rho-1} p^{N \frac{\rho}{2}}
\end{aligned}
$$

Hence from Assumption 2

$$
\Pi=O\left(\left(r p^{N}\right)^{1-\frac{\rho}{2}} D^{-\kappa}\right)+O\left(\frac{r D^{\rho-1} p^{N \frac{\rho}{2}}}{\left(r p^{N}\right)^{\frac{\rho}{2}}}\right) .
$$


Since $\rho>2$ and $\kappa>0$, hence

$$
\Pi=O\left(r^{1-\frac{\rho}{2}} D^{\rho-1}\right)
$$

which tends to zero for $D=o\left(r^{\frac{\rho-2}{2(\rho-1)}}\right)$.

Now we have

$$
S_{\mathbf{n}}=T(\mathbf{n}, x, 1)+\sum_{i=2}^{2^{N}} T(\mathbf{n}, x, i)
$$

$T(\mathbf{n}, x, 1)$ has been treated and without loss of generality, consider $T(\mathbf{n}, x, 2)$. Enumerate the random variables $U(2, \mathbf{n}, x, j)$ in the arbitrary manner and refer to them as $\widetilde{W}_{1}, \ldots, \widetilde{W}_{r}$. Now show that $\operatorname{Var}\left(\left(r p^{N}\right)^{\frac{-1}{2}} \sum \widetilde{W}_{m}\right) \rightarrow 0$.

$$
\begin{aligned}
\operatorname{Var}\left(\sum_{i=1}^{r} \widetilde{W}_{i}\right) & =\sum_{i=1}^{r} \operatorname{Var} \widetilde{W}_{i}+\sum_{i=1}^{r} \sum_{\substack{j=1 \\
i \neq j}}^{r} \operatorname{Cov}\left(\widetilde{W}_{i}, \widetilde{W}_{j}\right) \\
& =A_{1}+A_{2} .
\end{aligned}
$$

Since $Z_{t}$ is stationary, we have

$$
\begin{aligned}
\operatorname{Var} \tilde{W}_{i} & =\operatorname{Var}\left(\sum_{\substack{t_{k}=1 \\
k=1, \ldots, N}}^{p} Y_{\mathbf{t}, \mathbf{n}}(x)\right)^{2} \\
& =p^{N-1} q \operatorname{Var} Y_{\mathbf{t}, \mathbf{N}}(x)+\sum_{\substack{t_{k}=1 \\
k=1, \ldots, N-1}}^{p} \sum_{\substack{t_{N}=1 \\
t_{k}}}^{q} \sum_{\substack{j_{k}=1 \\
k=1, \ldots, N-1 \\
t_{k} \neq j_{k}, \text { for some } 1 \leq k \leq N .}}^{q} \sum_{j_{N}=1}^{q} E Y_{\mathbf{t}, \mathbf{n}}(x) Y_{\mathbf{j}, \mathbf{n}}(x) .
\end{aligned}
$$

However,

$$
\operatorname{Var} Y_{\mathbf{t}, \mathbf{n}}(x)=2 \sigma^{2}\left(\sum_{i=1}^{m} \sqrt{2}\left(u_{i} \cos \omega^{T} \mathbf{t}+v_{i} \sin \omega^{T} \mathbf{t}\right)\right)^{2}
$$

Note that as before

$$
\left|a_{t}\right|=\left|\sum_{i=1}^{m} \sqrt{2}\left(u_{i} \cos \omega^{T} \mathbf{t}+v_{i} \sin \omega^{T} \mathbf{t}\right)\right|<\left|\sum_{i=1}^{m}\left(\left|u_{i}\right|+\left|v_{i}\right|\right)\right|=C .
$$

Hence,

$$
E\left|Y_{\mathbf{t}, \mathbf{n}}(x) Y_{\mathbf{j}, \mathbf{n}}(x)\right| \leq C \alpha\|\mathbf{t}-\mathbf{j}\|^{\frac{\gamma-2}{\gamma}} .
$$

We have

$$
\begin{aligned}
\operatorname{Var} \tilde{W}_{i} \leq & 2 \sigma^{2} \sum_{\substack{t_{k}=1 \\
k=1, \ldots, N-1}}^{p} \sum_{\substack{t_{N}=1 \\
k=N}}^{q}\left(\sum_{i=1}^{m} \sqrt{2}\left(u_{i} \cos \omega^{T} t+v_{i} \sin \omega^{T} t\right)\right)^{2} \\
& +C \sum_{\substack{t_{k}=1 \\
k=1, \ldots, N-1}}^{p} \sum_{\substack{t_{N}=1 \\
t_{k} \neq j_{k}}}^{q} \sum_{\substack{j_{k}=1 \\
k=1, \ldots, N-1}}^{p} \sum_{j_{N}=1}^{q} \alpha(\|\mathbf{t}-\mathbf{j}\|)^{\frac{\gamma-2}{\gamma}}
\end{aligned}
$$




$$
\begin{aligned}
& \leq C p^{N-1} q\left(\sigma^{2}+\sum_{\substack{t_{k}=1 \\
k=1, \ldots, N}}^{p} \sum_{t_{N}=1}^{q} \alpha(\|\mathbf{t}\|)^{\frac{\gamma-2}{\gamma}}\right) \\
& \leq C p^{N-1} q\left(\sigma^{2}+\sum_{i=1}^{\infty} i^{N-1} \alpha(i)^{\frac{\gamma-2}{\gamma}}\right) .
\end{aligned}
$$

Let $I(2, n, x, j)=\left\{\mathbf{i}: j_{k}(p+q)+1 \leq i_{k} \leq j_{k}(p+q)+p, 1 \leq k \leq N-1, j_{N}(p+q)+p+1 \leq i_{N} \leq\right.$ $\left.\left(j_{N}+1\right)(p+q)\right\}$; hence $U(2, \mathbf{n}, x, \mathbf{j})$ is sum of $Y_{\mathbf{t}}$ in the sites $I(2, \mathbf{n}, x, j)$. If $\mathbf{j}$ and $\mathbf{j}$ ' are in two distinct sites, then $j_{k} \neq j_{k}^{\prime}$ for some $1 \leq k \leq N$ and $\left\|\mathbf{j}-\mathbf{j}^{\prime}\right\|>q$, since $p>q$.

We obtain

$$
\begin{aligned}
A_{2} & \leq C \sum_{\mathbf{t} \in \mathbf{I}_{\mathbf{n}}} \sum_{\mathbf{j} \in \mathbf{I}_{\mathbf{n}}} E\left|Y_{\mathbf{t}, \mathbf{n}}(x) Y_{\mathbf{j}, \mathbf{n}}(x)\right|, \quad\|\mathbf{t}-\mathbf{j}\|>q \\
& \leq C \widehat{\mathbf{n}} \sum_{\mathbf{i} \in \mathbf{I}_{\mathbf{n}}} \alpha(\|\mathbf{i}\|)^{\frac{\gamma-2}{\gamma}} \quad\|\mathbf{i}\|>q \\
& \leq C \widehat{\mathbf{n}} \sum_{i=q}^{\infty} i^{N-1} \alpha(i)^{\frac{\gamma-2}{\gamma}} .
\end{aligned}
$$

Hence

$$
\begin{aligned}
\operatorname{Var}\left(\sum_{m=1}^{r} \widetilde{W}_{m}\right) & \leq C r p^{N-1} q\left(\sigma^{2}+\sum_{i=1}^{\infty} i^{N-1} \alpha(\mathbf{i})^{\frac{\gamma-2}{\gamma}}\right)+C \widehat{\mathbf{n}} \sum_{i=q}^{\infty} i^{N-1} \alpha(\mathbf{i})^{\frac{\gamma-2}{\gamma}} \\
\left(r p^{N}\right)^{-1} \operatorname{Var}\left(\sum_{i=1}^{r} \widetilde{W}_{m}\right) & \leq C p^{-1} q\left(\sigma^{2}+\sum_{i=1}^{\infty} i^{N-1} \alpha(i)^{\frac{\gamma-2}{\gamma}}\right)+C\left(r p^{N}\right)^{-1} \widehat{\mathbf{n}} \sum_{i=q}^{\infty} i^{N-1} \alpha(i)^{\frac{\gamma-2}{\gamma}}, \\
\left(r p^{N}\right)^{-1} \operatorname{Var}\left(\sum_{m=1}^{r} \widetilde{W}_{m}\right) & \leq C p^{-1} q\left(\sigma^{2}+\sum_{i=1}^{\infty} i^{N-1} \alpha(i)^{\frac{\gamma-2}{\gamma}}\right)+C \sum_{i=q}^{\infty} i^{N-1} \alpha(i)^{\frac{\gamma-2}{\gamma}}
\end{aligned}
$$

Suppose that $\sum_{i=1}^{\infty} i^{N-1} \alpha(i)^{\frac{\gamma-2}{\gamma}}<\infty$. Then

$$
\left(r p^{N}\right)^{-1} \operatorname{Var}\left(\sum_{m=1}^{r} \widetilde{W}_{m}\right)=O(q / p)
$$

converges in probability to zero since $q<p$, hence the result.

(ii) In the following, we prove the result with $\lambda$ instead of $\lambda_{\mathbf{j}}$ and we make the substitution at the end of the proof. By the definition of $I_{\mathbf{n}, Z}(\lambda)$, we have

$$
I_{\mathbf{n}, Z}(\lambda)=\widehat{\mathbf{n}}^{-1} \sum_{\mathbf{i} \in \mathbf{I}_{\mathbf{n}}} \sum_{\mathbf{j} \in \mathbf{I}_{\mathbf{n}}} Z_{\mathbf{i}} Z_{\mathbf{j}} e^{-i \lambda^{T}(\mathbf{i}-\mathbf{j})} .
$$

Hence

$$
E\left(I_{\mathbf{n}, Z}(\lambda) I_{\mathbf{n}, Z}\left(\lambda^{\prime}\right)\right)=\widehat{\mathbf{n}}^{-2} \sum_{\mathbf{i} \in \mathbf{I}_{\mathbf{n}}} \sum_{\mathbf{j} \in \mathbf{I}_{\mathbf{n}}} \sum_{\mathbf{s} \in \mathbf{I}_{\mathbf{n}}} \sum_{\mathbf{u} \in \mathbf{I}_{\mathbf{n}}} E\left(Z_{\mathbf{i}} Z_{\mathbf{j}} Z_{\mathbf{s}} Z_{\mathbf{u}}\right) e^{-i \lambda^{T}(\mathbf{i}-\mathbf{j})} e^{-i \lambda^{\prime T}(\mathbf{s}-\mathbf{u})}
$$

Suppose that $E\left(Z_{\mathbf{i}}^{4}\right)=\theta \sigma^{4}$ and $\sum_{\mathbf{i} \in \mathbf{I}_{\mathbf{n}}} \alpha(\|\mathbf{i}\|)^{\frac{\gamma-2}{\gamma}}=\sum_{i=1}^{\infty} i^{N-1} \alpha(i)^{\frac{\gamma-2}{\gamma}}<\infty$. We will examine many cases: $1 . \mathbf{i}=\mathbf{j}=\mathbf{s}=\mathbf{u}$,

$$
T_{1}=\widehat{\mathbf{n}}^{-2} \sum_{\mathbf{i} \in \mathbf{I}_{\mathbf{n}}} E\left(Z_{\mathbf{i}}^{4}\right)=\widehat{\mathbf{n}}^{-1} \theta \sigma^{4} .
$$


2. $\mathbf{i}=\mathbf{j} \neq \mathbf{s}=\mathbf{u}$

$$
T_{2}=\widehat{\mathbf{n}}^{-2} \sum_{\mathbf{i} \in \mathbf{I}_{\mathbf{n}}} \sum_{\substack{\mathbf{s} \in \mathbf{I}_{\mathbf{n}} \\ \mathbf{i} \neq \mathbf{s}}} E\left(Z_{\mathbf{i}}^{2} Z_{\mathbf{s}}^{2}\right)
$$

Applying Davydov inequality, we have

$$
\left|\operatorname{Cov}\left(Z_{\mathbf{i}}^{2}, Z_{\mathbf{s}}^{2}\right)\right| \leq \frac{2 \gamma}{\gamma-2}\left\|Z_{\mathbf{i}}^{2}\right\|_{\gamma}\left\|Z_{\mathbf{s}}^{2}\right\|_{\gamma} 2 \alpha\left(\|\mathbf{i}-\mathbf{s}\|^{\frac{\gamma-2}{\gamma}} .\right.
$$

Hence

$$
\left|E\left(Z_{\mathbf{i}}^{2} Z_{\mathbf{s}}^{2}\right)\right|-\sigma^{4} \leq 2 C \alpha(\|\mathbf{i}-\mathbf{s}\|)^{\frac{\gamma-2}{\gamma}}
$$

Then

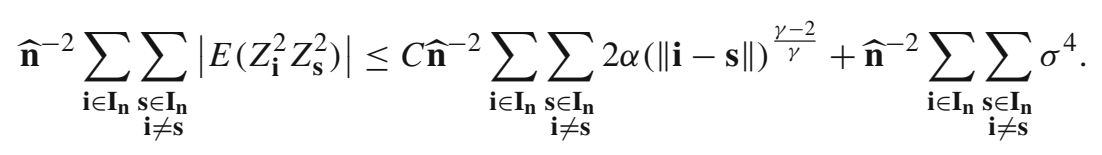

Hence

$$
\begin{aligned}
T_{2} & \leq C \widehat{\mathbf{n}}^{-2} \sum_{\substack{i_{k}=2 \\
k=1, \ldots, N}}^{n_{k}} \sum_{\substack{s_{k}=1 \\
\mathbf{i} \neq \mathbf{s}}}^{i_{k}-1} 2 \alpha(\|\mathbf{i}-\mathbf{s}\|)^{\frac{\gamma-2}{\gamma}}+\left(1-\frac{1}{\widehat{\mathbf{n}}}\right) \sigma^{4} \\
& =C \widehat{\mathbf{n}}^{-2} \sum_{\substack{i_{k}=2 \\
k=1, \ldots, N}}^{n_{k}} \sum_{v_{k}=1}^{i_{k}-1} \alpha(\|v\|)^{\frac{\gamma-2}{\gamma}}+\left(1-\frac{1}{\widehat{\mathbf{n}}}\right) \sigma^{4} .
\end{aligned}
$$

taking into account the convergence of the series of mixing coefficient, we have

$$
T_{2}=\left(1-\frac{1}{\widehat{\mathbf{n}}}\right) \sigma^{4}+O\left(\widehat{\mathbf{n}}^{-1}\right)
$$

$3-\mathbf{i}=\mathbf{u} \neq \mathbf{s}=\mathbf{j}$

$$
\begin{aligned}
& T_{3}=\widehat{\mathbf{n}}^{-2} \sum_{\mathbf{i} \in \mathbf{I}_{\mathbf{n}}} \sum_{\substack{\mathbf{s} \in \mathbf{I}_{\mathbf{n}} \\
\mathbf{i} \neq \mathbf{s}}} E\left(Z_{\mathbf{i}}^{2} Z_{\mathbf{s}}^{2}\right) e^{i\left(\lambda-\lambda^{\prime}\right)^{T}(\mathbf{i}-\mathbf{s})}
\end{aligned}
$$

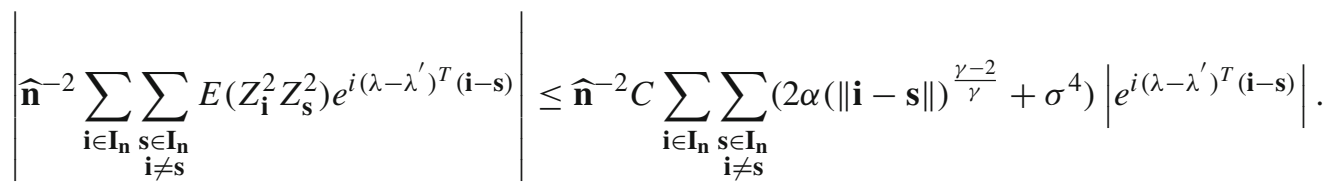

$$
\begin{aligned}
& T_{3}=\widehat{\mathbf{n}}^{-2} \sum_{\substack{i_{k}=2 \\
k=1, \ldots, N}}^{n_{k}} \sum_{s_{k}=1}^{i_{k}-1} 2 C \alpha(\|\mathbf{i}-\mathbf{s}\|)^{\frac{\gamma-2}{\gamma}}+\widehat{\mathbf{n}}^{-2} \sigma^{4}\left|\sum_{\mathbf{i} \in \mathbf{I}_{\mathbf{n}}} e^{-i\left(\lambda-\lambda^{\prime}\right)^{T} \mathbf{i}}\right|^{2}-\frac{1}{\mathbf{n}} \sigma^{4} \\
& T_{3}=C \widehat{\mathbf{n}}^{-2} \sum_{\substack{i_{k}=2 \\
k=1, \ldots, N}}^{n_{k}} \sum_{\nu_{k}=1}^{i_{k}-1} 2 \alpha(\|v\|)^{\frac{\gamma-2}{\gamma}}+\widehat{\mathbf{n}}^{-2} \sigma^{4}\left|\sum_{\mathbf{i} \in \mathbf{I}_{\mathbf{n}}} e^{-i\left(\lambda-\lambda^{\prime}\right)^{T} \mathbf{i}}\right|^{2}-\frac{1}{\widehat{n}} \sigma^{4}
\end{aligned}
$$

hence

$$
T_{3}=O\left(\widehat{\mathbf{n}}^{-1}\right)+\widehat{\mathbf{n}}^{-2} \sigma^{4}\left|\sum_{\mathbf{i} \in \mathbf{I}_{\mathbf{n}}} e^{i\left(\lambda-\lambda^{\prime}\right)^{T}} \mathbf{i}\right|^{2}-\frac{1}{\widehat{\hat{\mathbf{n}}}} \sigma^{4}
$$




$$
\begin{aligned}
& 4-\mathbf{i}=\mathbf{s} \neq \mathbf{j}=\mathbf{u} \\
& \left.T_{4}=\widehat{\mathbf{n}}^{-2} \sum_{\mathbf{i} \in \mathbf{I}_{\mathbf{n}}} \sum_{\substack{\mathbf{i} \in \mathbf{I}_{\mathbf{n}} \\
\mathbf{i} \neq \mathbf{j}}} E\left(Z_{\mathbf{i}}^{2} Z_{\mathbf{j}}^{2}\right) e^{i\left(\lambda+\lambda^{\prime}\right)^{T}(\mathbf{i}-\mathbf{j})}\right) \leq \widehat{\mathbf{n}}^{-2} \sum_{\mathbf{i} \in \mathbf{I}_{\mathbf{n}}} \sum_{\mathbf{j} \in \mathbf{I}_{\mathbf{n}}} C 2 \alpha(\|\mathbf{i}-\mathbf{s}\|)^{\frac{\gamma-2}{\gamma}}+\widehat{\mathbf{n}}^{-2} \sigma^{4}\left|\sum_{\mathbf{i} \in \mathbf{I}_{\mathbf{n}}} e^{i\left(\lambda+\lambda^{\prime}\right)^{T} \mathbf{i}}\right|^{2}-\frac{1}{\widehat{\mathbf{n}}} \sigma^{4}, \\
& T_{4} \leq C \widehat{\mathbf{n}}^{-2} \sum_{\substack{i_{k}=2 \\
k=1, \ldots, N}}^{n_{k}} \sum_{\nu_{k}=1}^{i_{k}-1} 2 \alpha(\|\nu\|)^{\frac{\gamma-2}{\gamma}}+\widehat{\mathbf{n}}^{-2} \sigma^{4}\left|\sum_{\mathbf{i} \in \mathbf{I}_{\mathbf{n}}} e^{i\left(\lambda+\lambda^{\prime}\right)^{T} \mathbf{i}}\right|^{2}-\frac{1}{\widehat{\mathbf{n}}} \sigma^{4}
\end{aligned}
$$

hence

$$
T_{4}=O\left(\widehat{\mathbf{n}}^{-1}\right)+\widehat{\mathbf{n}}^{-2} \sigma^{4}\left|\sum_{\mathbf{i} \in \mathbf{I}_{\mathbf{n}}} e^{i\left(\lambda+\lambda^{\prime}\right)^{T}} \mathbf{i}\right|^{2}-\frac{1}{\widehat{\widehat{n}}} \sigma^{4}
$$

$5-\mathbf{i}=\mathbf{j}, \mathbf{j} \neq \mathbf{s}, \mathbf{s} \neq \mathbf{u}$

$T_{5}=\left|\frac{3 !}{\widehat{\mathbf{n}}^{2}} \sum_{\substack{i_{k}=1 \\ k=1, \ldots, N}}^{n_{k}} \sum_{\substack{s_{k}=i_{k}+1 \\ k=1, \ldots, N}}^{n_{k}} \sum_{\substack{u_{k}=s_{k}+1 \\ k=1, \ldots, N}}^{n_{k}} E\left(Z_{\mathbf{i}}^{2} Z_{\mathbf{s}} Z_{\mathbf{u}}\right) e^{-i \lambda^{\prime T}(\mathbf{s}-\mathbf{u})}\right| \leq \frac{3 !}{\widehat{\mathbf{n}}^{2}} \sum_{\substack{i_{k}=1 \\ k=1, \ldots, N}}^{n_{k}} \sum_{\substack{s_{k}=i_{k}+1 \\ k=1, \ldots, N}}^{n_{k}}\left|\operatorname{cov}\left(Z_{\mathbf{i}}^{2}, Z_{\mathbf{s}} \sum_{\substack{u_{k}=s_{k}+1 \\ k=1, \ldots, N}}^{n_{k}} Z_{\mathbf{u}}\right)\right|$ $T_{5} \leq \frac{3 !}{\widehat{\mathbf{n}}^{2}} \sum_{\substack{i_{k}=1 \\ k=1, \ldots, N}}^{n_{k}} \sum_{\substack{s_{k}=i_{k}+1 \\ k=1, \ldots, N}}^{n_{k}} \alpha(\|\mathbf{i}-\mathbf{s}\|)=3 ! C \widehat{\mathbf{n}}^{-2} \sum_{\substack{i_{k}=2 \\ k=1, \ldots, N}}^{n_{k}} \sum_{\nu_{k}=1}^{i_{k}-1} 2 \alpha(\|v\|)$;

it follows, using the assumption on the mixing coefficient, that

$$
T_{5}=O\left(\widehat{\mathbf{n}}^{-1}\right) .
$$

The cases, $\mathbf{i}=\mathbf{s} \neq \mathbf{j} \neq \mathbf{u}, \mathbf{i}=\mathbf{u} \neq \mathbf{s} \neq \mathbf{j}, \mathbf{j}=\mathbf{s} \neq \mathbf{i} \neq \mathbf{u}, \mathbf{t}=\mathbf{u} \neq \mathbf{i} \neq \mathbf{s}$ and $\mathbf{s}=\mathbf{u} \neq \mathbf{i} \neq \mathbf{j}$, are treated in the same manner as $T_{5}$.

6- $\mathbf{i} \neq \mathbf{j} \neq \mathbf{s} \neq \mathbf{u}$

$$
\begin{gathered}
\left|T_{11}\right|=\left|\frac{1}{\widehat{\mathbf{n}}^{2}} \sum_{\mathbf{i} \in \mathbf{I}_{\mathbf{n}}} \sum_{\mathbf{j} \in \mathbf{I}_{\mathbf{n}}} \sum_{\mathbf{s} \in \mathbf{I}_{\mathbf{n}}} \sum_{\mathbf{u} \in \mathbf{I}_{\mathbf{n}}} E\left(Z_{\mathbf{i}} Z_{\mathbf{j}} Z_{\mathbf{s}} Z_{\mathbf{u}}\right) e^{i \lambda^{T}(\mathbf{i}-\mathbf{j})} e^{-i \lambda^{\prime T}(\mathbf{s}-\mathbf{u})}\right|, \\
\left|T_{11}\right| \leq \frac{4 !}{\widehat{\mathbf{n}}^{2}} \sum_{\substack{i_{k}=1 \\
k=1, \ldots, N}}^{n_{k}} \sum_{\substack{j_{k}=i_{k}+1 \\
k=1, \ldots, N}}^{n_{k}} \operatorname{Cov}\left(Z_{\mathbf{i}}, Z_{\mathbf{j}} \sum_{\substack{s_{k}=j_{k}+1 \\
k=1, \ldots, N}}^{n_{k}} \sum_{\substack{u_{k}=s_{k}+1 \\
k=1, \ldots, N}}^{n_{k}} Z_{\mathbf{s}} Z_{\mathbf{u}}\right) \mid, \\
T_{11} \leq \frac{4 !}{\widehat{\mathbf{n}}^{2}} \sum_{\substack{i_{k}=1 \\
k=1, \ldots, N}}^{n_{k}} \sum_{\substack{j_{k}=i_{k}+1 \\
k=1, \ldots, N}}^{n_{k}} \alpha(\|\mathbf{i}-\mathbf{j}\|) \leq \frac{4 !}{\widehat{\mathbf{n}}^{2}} \sum_{\substack{i_{k}=2 \\
k=1, \ldots, N}}^{n_{k}} \sum_{\nu_{k}=1}^{i_{k}-1} 2 \alpha(\|v\|) .
\end{gathered}
$$

Hence

$$
T_{11}=O\left(\widehat{\mathbf{n}}^{-1}\right) .
$$

$7-\mathbf{i} \neq \mathbf{j}=\mathbf{s}=\mathbf{u}$

$$
T_{12}=\left|\widehat{\mathbf{n}}^{-2} \sum_{\mathbf{i} \in \mathbf{I}_{\mathbf{n}}} \sum_{\substack{\mathbf{j} \in \mathbf{I}_{\mathbf{n}} \\ \mathbf{i} \neq \mathbf{j}}} E\left(Z_{\mathbf{i}} Z_{\mathbf{j}}^{3}\right) e^{-i \lambda^{T}(\mathbf{i}-\mathbf{j})}\right|
$$




$$
\begin{aligned}
& \leq\left|2 \widehat{\mathbf{n}}^{-2} \sum_{\substack{i_{k}=1 \\
k=1, \ldots, N}}^{n_{k}} \sum_{\substack{j_{k}=i_{k}+1 \\
\mathbf{i} \neq \mathbf{j}}}^{n_{k}} \operatorname{Cov}\left(Z_{\mathbf{i}}, Z_{\mathbf{j}}^{3}\right)\right| \\
& \leq 2 \widehat{\mathbf{n}}^{-2} \sum_{\substack{i_{k}=1 \\
k=1, \ldots, N}}^{n_{k}} \sum_{j_{k}=i_{k}+1}^{n_{k}} \alpha(\|\mathbf{i}-\mathbf{s}\|) \leq 2 \widehat{\mathbf{n}}^{-2} \sum_{\substack{i_{k}=2 \\
k=1, \ldots, N}}^{n_{k}} \sum_{\nu_{k}=1}^{i_{k}-1} 2 \alpha(\|v\|) .
\end{aligned}
$$

It follows that

$$
T_{12}=O\left(\widehat{\mathbf{n}}^{-1}\right) .
$$

We obtain the same results for the following cases $\mathbf{j} \neq \mathbf{s}=\mathbf{i}=\mathbf{u}, \mathbf{s} \neq \mathbf{u}=\mathbf{i}=\mathbf{j}, \mathbf{u} \neq \mathbf{s}=\mathbf{j}=\mathbf{i}$.

Taking into account all $T_{i}$, we have

$$
\begin{aligned}
\operatorname{Cov}\left(I_{\mathbf{n}, Z}(\omega), I_{\mathbf{n}, Z}\left(\omega^{\prime}\right)\right) & =E\left(I_{\mathbf{n}, Z}(\lambda) I_{\mathbf{n}, Z}\left(\lambda^{\prime}\right)\right)-E I_{\mathbf{n}, Z}(\lambda) E I_{\mathbf{n}, Z}\left(\lambda^{\prime}\right) \\
& =\sum T_{i}-\sigma^{4} .
\end{aligned}
$$

Since

$$
\begin{aligned}
E I_{\mathbf{n}, Z}(\omega) & =\widehat{\mathbf{n}}^{-1} \sum_{\mathbf{i} \in \mathbf{I}_{\mathbf{n}}} \sum_{\mathbf{j} \in \mathbf{I}_{\mathbf{n}}} E\left(Z_{\mathbf{i}} Z_{\mathbf{j}}\right) e^{-i \lambda^{T}(\mathbf{i}-\mathbf{j})} \\
& =\widehat{\mathbf{n}}^{-1} \sum_{\mathbf{i} \in \mathbf{I}_{\mathbf{n}}} E\left(Z_{\mathbf{i}}^{2}\right)+\widehat{\mathbf{n}}^{-1} \sum_{\substack{i_{k}=2 \\
k=1, \ldots, N}}^{n_{k}} \sum_{\nu_{k}=1}^{i_{k}-1}(2 \alpha(\|\imath\|) \\
& =\sigma^{2}+\widehat{\mathbf{n}}^{-1} \sum_{\substack{i_{k}=2 \\
k=1, \ldots, N}}^{n_{k}} \alpha(\|\mathbf{i}\|)^{\frac{\gamma}{\gamma-2}} \\
& =\sigma^{2}+O\left(\widehat{\mathbf{n}}^{-1}\right) .
\end{aligned}
$$

Hence

$$
\begin{aligned}
\operatorname{Cov}\left(I_{\mathbf{n}, Z}(\lambda), I_{\mathbf{n}, Z}\left(\lambda^{\prime}\right)\right)= & \widehat{\mathbf{n}}^{-1} \theta \sigma^{4}+\left(1-\frac{1}{\widehat{\mathbf{n}}}\right) \sigma^{4}+\widehat{\mathbf{n}}^{-2} \sigma^{4}\left|\sum_{\mathbf{s} \in \mathbf{I}_{\mathbf{n}}} e^{i\left(\lambda-\lambda^{\prime}\right)^{T} \mathbf{s}}\right|^{2} \\
& -\frac{2}{\widehat{\mathbf{n}}} \sigma^{4}+\widehat{\mathbf{n}}^{-2} \sigma^{4}\left|\sum_{\mathbf{s} \in \mathbf{I}_{\mathbf{n}}} e^{i\left(\lambda+\lambda^{\prime}\right)^{T} \mathbf{s}}\right|^{2}-\sigma^{4}+O\left(\widehat{\mathbf{n}}^{-1}\right) \\
= & \widehat{\mathbf{n}}^{-1}(\theta-3) \sigma^{4} \\
& +\widehat{\mathbf{n}}^{-2} \sigma^{4}\left[\left|\sum_{\mathbf{s} \in \mathbf{I}_{\mathbf{n}}} e^{i\left(\lambda+\lambda^{\prime}\right)^{T} \mathbf{s}}\right|^{2}+\left|\sum_{\mathbf{s} \in \mathbf{I}_{\mathbf{n}}} e^{i\left(\lambda-\lambda^{\prime}\right)^{T} \mathbf{s}}\right|^{2}\right]+O\left(\widehat{\mathbf{n}}^{-1}\right) .
\end{aligned}
$$

Hence for $\lambda_{\mathbf{j}}=\left(\lambda_{\mathbf{j} 1}, \ldots, \lambda_{\mathbf{j} N}\right)$

$$
\begin{aligned}
& \operatorname{Cov}\left(I_{\mathbf{n}, Z}\left(\lambda_{\mathbf{j}}\right), I_{\mathbf{n}, Z}\left(\lambda_{\mathbf{l}}\right)\right) \\
& =\left\{\begin{array}{l}
\widehat{\mathbf{n}}^{-1}(\theta-3) \sigma^{4}+2 \sigma^{4}+O\left(\widehat{\mathbf{n}}^{-1}\right) \text { if } \lambda_{\mathbf{j}}=\lambda_{\mathbf{I}} \text { and } \lambda_{\mathbf{j} i}=0 \text { or } \pi, \forall i=1 \ldots, N \\
\widehat{\mathbf{n}}^{-1}(\theta-3) \sigma^{4}+2 \sigma^{4}+O\left(\widehat{\mathbf{n}}^{-1}\right) \text { if } \lambda_{\mathbf{j}}=\lambda_{\mathbf{l}} \text { and } 0<\lambda_{\mathbf{j} i}<\pi, \forall i=1, \ldots, N \\
\widehat{\mathbf{n}}^{-1}(\theta-3) \sigma^{4}+O\left(\widehat{\mathbf{n}}^{-1}\right) \quad \text { if } \lambda_{\mathbf{j}} \neq \lambda_{\mathbf{l}} .
\end{array}\right.
\end{aligned}
$$


In the following theorem, we establish the relation between the periodograms of $\left\{X_{\mathbf{t}}\right\}$ and $\left\{Z_{\mathbf{t}}\right\}$.

Let $a\left(e^{-i \omega}\right)=\sum_{\mathbf{j} \in \mathbb{Z}^{N}} a_{\mathbf{j}} \exp \left(-i \omega^{T} \mathbf{j}\right)$, where $\omega=\left(\omega_{1}, \ldots, \omega_{N}\right)$.

Theorem 2 Under Assumptions 1 and 2 , and if $\omega \in[0, \pi]^{N}$, we can write

$$
I_{\mathbf{n}, X}(\omega)=\left(\left|a\left(e^{-i g(\mathbf{n}, \omega)}\right)\right|^{2}\right) I_{\mathbf{n}, Z}(\omega)+R_{\mathbf{n}}(g(\mathbf{n}, \omega)),
$$

where $\sup _{\omega}\left|R_{\mathbf{n}}(\omega)\right| \rightarrow 0$. If in addition $\sum_{\mathbf{j} \in \mathbb{Z}^{N}}\left|a_{\mathbf{j}}\right||\hat{\mathbf{j}}|^{\frac{1+\delta}{2}}<\infty$, then

$$
\sup _{\omega} E\left|R_{\mathbf{n}}(\omega)\right|^{2}=0\left(\widehat{\mathbf{n}}^{-1}\right) .
$$

Proof Let $J_{X}(\omega)$ and $J_{Z}(\omega)$ the discrete Fourier transform of $\left\{X_{\mathbf{t}}\right\}$ and $\left\{Z_{\mathbf{t}}\right\}$, respectively. Then

$$
\begin{aligned}
J_{X}(\omega) & =\widehat{\mathbf{n}}^{\frac{-1}{2}} \sum_{\mathbf{t} \in \mathbf{I}_{\mathbf{n}}} X_{\mathbf{t}} e^{-i \omega^{T} \mathbf{t}}=\widehat{\mathbf{n}}^{-\frac{1}{2}} \sum_{\mathbf{j} \in \mathbb{Z}^{N}} a_{\mathbf{j}} e^{-i \omega^{T} \mathbf{j}} \sum_{\mathbf{t} \in \mathbf{I}_{\mathbf{n}}} Z_{\mathbf{t}-\mathbf{j}} e^{-i \omega^{T}(\mathbf{t}-\mathbf{j})} \\
& =\widehat{\mathbf{n}}^{\frac{-1}{2}} \sum_{\mathbf{j} \in \mathbb{Z}^{N}} a_{\mathbf{j}} e^{-i \omega^{T} \mathbf{j}} \sum_{\substack{t_{k}=1-j_{k} \\
k=1, \ldots, N}}^{n_{k}-j_{k}} Z_{\mathbf{t}} e^{-i \omega^{T} \mathbf{t}} \\
& =\widehat{\mathbf{n}}^{\frac{-1}{2}} \sum_{\mathbf{j} \in \mathbb{Z}^{N}} a_{\mathbf{j}} e^{-i \omega^{T} \mathbf{j}}\left(\sum_{\mathbf{t} \in \mathbf{I}_{\mathbf{n}}} Z_{\mathbf{t}} e^{-i \omega^{T} \mathbf{t}}+\sum_{\substack{t_{k}=1-j_{k} \\
k=1, \ldots, N}}^{n_{k}-j_{k}} Z_{\mathbf{t}} e^{-i \omega^{T} \mathbf{t}}-\sum_{\mathbf{t} \in \mathbf{I}_{\mathbf{n}}} Z_{\mathbf{t}} e^{-i \omega^{T} \mathbf{t}}\right) .
\end{aligned}
$$

Note by

$$
S_{\mathbf{n}, j}=\sum_{\substack{t_{k}=1-j_{k} \\ k=1, \ldots, N}}^{n_{k}-j_{k}} Z_{\mathbf{t}} e^{-i \omega^{T} \mathbf{t}}-\sum_{\substack{t_{k}=1 \\ k=1, \ldots, N}}^{n_{k}} Z_{\mathbf{t}} e^{-i \omega^{T} \mathbf{t}}
$$

and

$$
Y_{\mathbf{n}}(\omega)=\widehat{\mathbf{n}}^{\frac{-1}{2}} \sum_{\mathbf{j} \in \mathbb{Z}^{N}} a_{\mathbf{j}} e^{-i \omega^{T} \mathbf{j}} S_{\mathbf{n}, \mathbf{j}}
$$

Then

$$
J_{X}(\omega)=\left(\widehat{\mathbf{n}}^{\frac{-1}{2}} \sum_{\mathbf{j} \in \mathbb{Z}^{N}} a_{\mathbf{j}} e^{-i \omega^{T} \mathbf{j}}\right) J_{Z}(\omega)+Y_{\mathbf{n}}(\omega) .
$$

Note that we have two cases:

In the first case, that is, $\left|j_{k}\right|<n_{k}$, we have, by Theorem 2.1 of [10] and under Assumptions 1 and 2 with $\rho=2$,

$$
\begin{aligned}
E\left|S_{n \mathbf{j}}\right|^{2} & \leq 2^{N} \widehat{\mathbf{j}}\left|M_{2 \gamma}^{2} \sum_{t=P}^{\infty} t^{N-1} \alpha(t)^{\frac{\gamma-2}{\gamma}}+2^{N} \widehat{\mathbf{j}}\right| P C_{\theta} \\
& \leq 2^{N} P^{-\kappa}|\widehat{\mathbf{j}}| P^{\kappa} M_{2 \gamma}^{2} \sum_{t=P}^{\infty} t^{N-1} \alpha(t)^{\frac{\gamma-2}{\gamma}}+2^{N}|\widehat{\mathbf{j}}| P C_{\theta} . \\
& \leq 2^{N}|\hat{\mathbf{j}}| P\left[P^{-\kappa-1} P^{\kappa} M_{2 \gamma}^{2} \sum_{t=P}^{\infty} t^{N-1} \alpha(t)^{\frac{\gamma-2}{\gamma}}+C_{\theta}\right] .
\end{aligned}
$$


Since $0<P<\widehat{\mathbf{n}}, P \rightarrow \infty$ and under Assumption 2, we have $P^{\kappa} \sum_{t=P}^{\infty} t^{N-1} \alpha(t)^{\frac{\gamma-2}{\gamma}} \rightarrow 0$, then

$$
E\left|S_{n \mathbf{j}}\right|^{2} \leq C|\hat{\mathbf{j}}| P .
$$

In the second case, there is $\widehat{\mathbf{n}}^{*}$ terms, in the same manner as before, we have

$$
E\left|S_{n \mathbf{j}}\right|^{2}=O\left(\widehat{\mathbf{n}}^{*} P\right)
$$

such that $P=O\left(\widehat{\mathbf{n}}^{* \delta}\right)$. Hence

$$
E\left|S_{n \mathbf{j}}\right|^{2}=O\left(\widehat{\mathbf{n}}^{*(1+\delta)}\right) .
$$

Since $\widehat{\mathbf{n}}^{*} \leq 2^{N} \widehat{\mid \mathbf{j}} \mid$, then

$$
E\left|S_{n \mathbf{j}}\right|^{2}=O\left(|\hat{\mathbf{j}}|^{1+\delta}\right)
$$

then

$$
E\left(Y_{\mathbf{n}}^{2}(\omega)\right) \leq\left(\widehat{\mathbf{n}}^{\frac{-1}{2}} \sum_{\mathbf{j} \in \mathbb{Z}^{N}}\left|a_{\mathbf{j}}\right| e^{-i \omega^{T} \mathbf{j}}\left(E\left(S_{\mathbf{n}, \mathbf{j}}^{2}\right)\right)^{\frac{1}{2}}\right)^{2}
$$

and

$$
E\left(Y_{\mathbf{n}}^{2}(\omega)\right) \leq 2 \sigma^{2}\left(\widehat{\mathbf{n}}^{\frac{-1}{2}} \sum_{\mathbf{j} \in \mathbb{Z}^{N}}\left|a_{\mathbf{j}}\right|\left(C_{\theta}|\widehat{\mathbf{j}}|\right)^{\frac{1+\delta}{2}}\right)^{2}
$$

Since

$$
\sum_{\mathbf{j} \in \mathbb{Z}^{N}}\left|a_{\mathbf{j}}\right|(|\widehat{\mathbf{j}}|)^{\frac{1+\delta}{2}}<\infty
$$

Then,

$$
E\left(Y_{\mathbf{n}}^{2}(\omega)\right) \rightarrow 0, \quad \text { as } \quad \mathbf{n} \rightarrow \infty
$$

Therefore, since we have

$$
R_{\mathbf{n}}(\omega)=a\left(e^{-i \omega}\right) J_{Z}(\omega) Y_{\mathbf{n}}(-\omega)+a\left(e^{-i \omega}\right) J_{Z}(-\omega) Y_{\mathbf{n}}(\omega)+\left|Y_{\mathbf{n}}(\omega)\right|^{2},
$$

and recalling that $I_{\mathbf{n}, X}\left(\omega_{k}\right)=J_{X}\left(\omega_{k}\right) J_{X}\left(-\omega_{k}\right)$, application of the Cauchy-Shwartz inequality gives in the same way as in [6] page $347, \max _{\omega \in[0, \pi]^{N}} E\left|R_{\mathbf{n}}(\omega)\right| \rightarrow 0$, as $\mathbf{n} \rightarrow \infty$.

In the first case, that is, $\left|j_{k}\right|<n_{k}$, by Assumptions 1 and 2 with $\rho=4$ and applying Theorem 2.1 in [10] again, we have

$$
\begin{aligned}
E\left|S_{\mathbf{n j}}\right|^{4} & \leq 2^{2 N}|\hat{\mathbf{j}}|^{2} M_{2 \gamma}^{4} \sum_{t=P}^{\infty} t^{2 N-1} \alpha(t)^{\frac{\gamma-2}{\gamma}}+2^{N}|\hat{\mathbf{j}}| P^{3} C_{\theta}+2^{2 N}|\hat{\mathbf{j}}|^{2} P^{3} C_{\theta}^{2} \\
& \leq 2^{2 N} P^{-\kappa}|\widehat{\mathbf{j}}|^{2} M_{2 \gamma}^{4}\left(P^{\kappa} \sum_{t=P}^{\infty} t^{2 N-1} \alpha(t)^{\frac{\gamma-2}{\gamma}}\right)+2^{N} P^{3}|\hat{\mathbf{j}}| C_{\theta}+2^{2 N} P^{3}|\hat{\mathbf{j}}|^{2} C_{\theta}^{2} \\
& \leq 2^{2 N}|\hat{\mathbf{j}}|^{2} P^{3}\left[P^{-\kappa-3} M_{2 \gamma}^{4}\left(P^{\kappa} \sum_{t=P}^{\infty} t^{2 N-1} \alpha(t)^{\frac{\gamma-2}{\gamma}}\right)+2^{-N}|\hat{\mathbf{j}}|^{-1} C_{\theta}+C_{\theta}^{2}\right]
\end{aligned}
$$


Since $0<P<\widehat{\mathbf{n}}, P \rightarrow \infty$ and under Assumption 2, we have $P^{\kappa} \sum_{t=P}^{\infty} t^{2 N-1} \alpha(t)^{\frac{\gamma-2}{\gamma}} \rightarrow 0$, hence

$$
E\left|S_{\mathbf{n j}}\right|^{4} \leq 2^{N}|\hat{\mathbf{j}}| P^{3} C_{\theta}+2^{2 N}|\hat{\mathbf{j}}|^{2} P^{3} C_{\theta}^{2} .
$$

The second case is treated in the same manner, that is, there is $\widehat{\mathbf{n}}^{*}$ terms, as in the first case we have

$$
E\left|S_{\mathbf{n j}}\right|^{4} \leq \widehat{\mathbf{n}}^{*} P^{3} C_{\theta}+\left(\widehat{\mathbf{n}}^{*}\right)^{2} P^{3} C_{\theta}^{2} .
$$

Since $\widehat{\mathbf{n}}^{*} \leq 2^{N} \widehat{\mathbf{j}} \mid$, then we find again the same result as in the first case.

$$
E\left|S_{\mathbf{n} \mathbf{j}}\right|^{4} \leq 2^{N}|\hat{\mathbf{j}}| P^{3} C_{\theta}+2^{2 N}|\hat{\mathbf{j}}|^{2} P^{3} C_{\theta}^{2} .
$$

Then in both cases, we have

$$
E\left(Y_{\mathbf{n}}^{4}(\omega)\right) \leq \widehat{\mathbf{n}}^{-2}\left(\sum_{\mathbf{j} \in \mathbb{Z}^{N}}\left|a_{\mathbf{j}}\right|\left(2^{N} P^{3}\left(|\hat{\mathbf{j}}| C_{\theta}+2^{2 N}|\widehat{\mathbf{j}}|^{2} C_{\theta}^{2}\right)\right)^{\frac{1}{4}}\right)^{4} .
$$

Set $P=O\left(\widehat{\mathbf{j}}^{2 \delta} \mid\right)$,

$$
\begin{aligned}
E\left(Y_{\mathbf{n}}^{4}(\omega)\right) & \leq C \widehat{\mathbf{n}}^{-2}\left(\sum_{\mathbf{j} \in \mathbb{Z}^{N}}\left|a_{\mathbf{j}}\right|\left(|\hat{\mathbf{j}}|^{2(\delta+1)}\right)^{\frac{1}{4}}\right)^{4} \\
& \leq C \widehat{\mathbf{n}}^{-2}\left(\sum_{\mathbf{j} \in \mathbb{Z}^{N}}\left|a_{\mathbf{j}}\right||\hat{\mathbf{j}}|^{\frac{1+\delta}{2}}\right)^{4} .
\end{aligned}
$$

Since $\sum_{\mathbf{j} \in \mathbb{Z}^{N}}\left|a_{\mathbf{j}}\right||\hat{\mathbf{j}}|^{\frac{1+\delta}{2}}<\infty$, hence

$$
E\left(Y_{\mathbf{n}}^{4}(\omega)\right)=0\left(\widehat{\mathbf{n}}^{-2}\right) .
$$

As in [6] page 347, by applying Cauchy-Shwartz inequality and results on the covariance of the periodogram of innovations in Theorem 1, we obtain

$$
\max _{\omega \in[0, \pi]^{N}} E\left|R_{\mathbf{n}}(\omega)\right|^{2}=O\left(\widehat{\mathbf{n}}^{-1}\right)
$$

The following theorem provides the asymptotic properties of the periodogram of $\left(X_{\mathfrak{t}}\right)$.

Theorem 3 Let $\sum_{\mathbf{j} \in \mathbb{Z}^{N}}\left|a_{\mathbf{j}}\right|<\infty$. Assume that for $\gamma>2$, and $m_{\gamma}>0, E\left(\left|Z_{\mathbf{t}}\right|^{2 \gamma}\right)=m_{\gamma}, \forall \mathbf{t} \in \mathbb{Z}^{N}$ and $\sum_{i} i^{N-1} \alpha(i)^{\frac{\gamma-2}{\gamma}}<\infty$.

i) Let $\lambda_{1} \leq \cdots \leq \lambda_{m} \in[0, \pi]^{N}$ be the ordered sequence of $\omega_{\mathbf{k}^{1}}, \ldots, \omega_{\mathbf{k}^{m}}$ defined above. If $\alpha(u) \simeq u^{-\beta}$ for some $\beta>\frac{\gamma N}{\gamma-2}$, then for $\lambda_{1}, \ldots, \lambda_{m} \in[0, \pi]^{N}$, the random vector $\left(I_{\mathbf{n}, X}\left(\lambda_{1}\right), \ldots, I_{\mathbf{n}, X}\left(\lambda_{m}\right)\right)$ converges as $\mathbf{n} \rightarrow \infty$ to a vector of independent and exponentially distributed random variables, where the $i^{\text {th }}$ element is of mean equal to $2 \pi f_{X}\left(\lambda_{i}\right), i=1, \ldots, m$.

ii) If $E Z_{1}^{4}=\theta \sigma^{4}<\infty$, then

$$
\begin{aligned}
& \operatorname{Cov}\left(I_{\mathbf{n}, X}\left(\lambda_{\mathbf{j}}\right), I_{\mathbf{n}, X}\left(\lambda_{\mathbf{l}}\right)\right) \\
& =\left\{\begin{array}{l}
2(2 \pi)^{2} f_{X}^{2}\left(\lambda_{j}\right)+O\left(\widehat{\mathbf{n}}^{-\frac{1}{2}}\right) \text { if } \lambda_{\mathbf{j}}=\lambda_{\mathbf{I}} \text { and } \lambda_{\mathbf{j}, k}=0 \text { or } \pi, \forall k=1, \ldots, N \\
(2 \pi)^{2} f_{X}^{2}\left(\lambda_{\mathbf{j}}\right)+O\left(\widehat{\mathbf{n}}^{-\frac{1}{2}}\right) \text { if } \lambda_{\mathbf{j}}=\lambda_{\mathbf{l}} \text { and } 0<\lambda_{\mathbf{j}, k}<\pi, \forall k=1, \ldots, N \\
O\left(\widehat{\mathbf{n}}^{-\frac{1}{2}}\right) \text { if } \lambda_{\mathbf{j}} \neq \lambda_{\mathbf{I}} .
\end{array}\right.
\end{aligned}
$$


Proof We have from Theorem 2

$$
I_{\mathbf{n}, X}(\lambda)=\left|a e^{-i g(\mathbf{n}, \lambda)}\right|^{2} I_{\mathbf{n}, Z}(\lambda)+R_{\mathbf{n}}(g(\mathbf{n}, \lambda)),
$$

where $a\left(e^{-i \lambda}\right)=\sum_{\mathbf{j} \in \mathbb{Z}^{N}} a_{\mathbf{j}} e^{-i \lambda^{T} \mathbf{j}}, \lambda \in[0, \pi]^{N}, \sup _{\lambda}\left|R_{\mathbf{n}}(g(\mathbf{n}, \lambda))\right| \rightarrow 0$ and $f_{Z}(\lambda)=\frac{\sigma^{2}}{(2 \pi)^{N}}$.

It is well known that the spectral density of $\left\{X_{\mathbf{t}}\right\}$ is related to the spectral density of $\left\{Z_{\mathbf{t}}\right\}$ by

$$
f_{X}(\lambda)=\left|a\left(e^{-i \lambda}\right)\right|^{2} f_{Z}(\lambda), \lambda \in[-\pi, \pi]^{N} .
$$

Hence

$$
\left|a\left(e^{-i \lambda}\right)\right|^{2}=\frac{f_{X}(\lambda)}{f_{Z}(\lambda)}
$$

Then

$$
\begin{aligned}
I_{\mathbf{n}, X}(\lambda) & =\frac{f_{X}(g(n, \lambda))}{f_{Z}(g(n, \lambda))} I_{\mathbf{n}, Z}(\lambda)+R_{\mathbf{n}}(g(\mathbf{n}, \lambda)), \\
& =\frac{(2 \pi)^{N}}{\sigma^{2}} f_{X}(g(\mathbf{n}, \lambda)) I_{\mathbf{n}, Z}(\lambda)+R_{\mathbf{n}}(g(\mathbf{n}, \lambda)) .
\end{aligned}
$$

Since $f_{X}(g(\mathbf{n}, \lambda)) \rightarrow f_{X}(\lambda)$ and $R_{\mathbf{n}}(g(\mathbf{n}, \lambda)) \rightarrow 0$ in probability, the result of the first part of the theorem is obtained from Theorem 1 .

(ii) The proof is similar to the proof of Theorem 2.2 in [5], then omitted. It suffices to see that

$$
\begin{aligned}
\operatorname{Var}\left(I_{n ; X}\left(\lambda_{k}\right)\right)= & \left(\frac{(2 \pi)^{N}}{\sigma^{2}}\right)^{2} f_{X}^{2}\left(\lambda_{k}\right) \operatorname{Var} I_{n, Z}\left(\lambda_{k}\right)+\operatorname{Var}\left(R_{n}\left(\lambda_{k}\right)\right) \\
& +\left(\frac{(2 \pi)^{N}}{\sigma^{2}}\right) f_{X}\left(\lambda_{k}\right) \operatorname{Cov}\left(I_{n, Z}\left(\lambda_{k}\right), R_{n}\left(\lambda_{k}\right)\right)
\end{aligned}
$$

We have $\operatorname{Var}\left(R_{\mathbf{n}}\left(\lambda_{\mathbf{k}}\right)\right) \leq E\left(R_{\mathbf{n}}\left(\lambda_{\mathbf{k}}\right)\right)^{2}$ and $\operatorname{Var}\left(I_{\mathbf{n}, Z}\left(\lambda_{\mathbf{k}}\right)\right)$ is bounded uniformly in $\lambda_{\mathbf{k}}$. Using CauchySchwartz inequality and since from Theorems 2 and 1 , respectively, we have $\sup E\left|R_{\mathbf{n}}(\lambda)\right|^{2}=O\left(\widehat{\mathbf{n}}^{-1}\right)$ and $\operatorname{Var}\left(I_{\mathbf{n}, Z}\left(\lambda_{\mathbf{k}}\right)\right)=O\left(\widehat{\mathbf{n}}^{-1}\right)$, and we have

$$
\operatorname{Cov}\left(I_{\mathbf{n}, Z}\left(\lambda_{\mathbf{k}}\right), R_{\mathbf{n}}\left(\lambda_{k}\right)\right)=O\left(\widehat{\mathbf{n}}^{-\frac{1}{2}}\right)
$$

Similar arguments give

$$
\operatorname{Cov}\left(I_{\mathbf{n}, X}\left(\lambda_{\mathbf{k}}\right), I_{\mathbf{n}, X}\left(\lambda_{\mathbf{k}}^{\prime}\right)\right)=O\left(\widehat{\mathbf{n}}^{-\frac{1}{2}}\right), \text { for } \lambda_{\mathbf{k}} \neq \lambda_{\mathbf{k}}^{\prime}
$$

Then from Theorem 1, we have

$$
\begin{aligned}
& \operatorname{Var}\left(I_{\mathbf{n}, X}\right)\left(\lambda_{\mathbf{k}}\right) \\
& \quad=\left\{\begin{array}{l}
2(2 \pi)^{2 N} f_{X}^{2}\left(\lambda_{\mathbf{k}}\right)+O\left(\widehat{\mathbf{n}}^{-\frac{1}{2}}\right) \text { if } \lambda_{\mathbf{j}}=\lambda_{\mathbf{I}} \text { and } \lambda_{\mathbf{j} i}=0 \text { or } \pi, \forall i=1 \ldots, N \\
(2 \pi)^{2 N} f_{X}^{2}\left(\lambda_{\mathbf{k}}\right)+O\left(\widehat{\mathbf{n}}^{-\frac{1}{2}}\right) \text { if } \lambda_{\mathbf{j}}=\lambda_{\mathbf{I}} \text { and } 0<\lambda_{\mathbf{j} i}<\pi, \forall i=1 \text { to } N \text { and } \sum w_{\mathbf{j} i} s_{k} \neq k \pi
\end{array}\right] .
\end{aligned}
$$




\section{Convergence of the smoothed periodogram}

Since the periodogram estimator is not consistent, we introduce a linear filter and obtain a smoother periodogram. In this section, we study the convergence properties of this smoother periodogram estimator. Let $\left(X_{\mathbf{t}}, \mathbf{t} \in \mathbb{Z}^{N}\right)$ be the linear process defined above, consider the following class of estimators

$$
\widehat{f}\left(\omega_{\mathbf{j}}\right)=(2 \pi)^{-N} \sum_{k_{r},\left|k_{r}\right| \leq l_{n_{r}} r=1, \ldots N} W_{\mathbf{n}}(\mathbf{k}) I_{\mathbf{n}, X}\left(\omega_{\mathbf{j}+\mathbf{k}}\right),
$$

such that $\omega_{\mathbf{j}}=\left(\frac{2 \pi j_{1}}{n_{1}}, \ldots, \frac{2 \pi j_{N}}{n_{N}}\right)$ and where $l_{n_{r}} \rightarrow \infty, n_{r} \rightarrow \infty$ and $l_{n_{r}}<c n_{r}$ for some $c \in[0,1] .\left(W_{\mathbf{n}}, \mathbf{n} \in\right.$ $\left.\mathbb{N}^{N}\right)$ is a sequence of symmetric weight functions such that $W_{\mathbf{n}}(\mathbf{k})$ are obtained as product of one-dimensional windows: $W_{\mathbf{n}}(\mathbf{k})=W_{n_{1}}\left(k_{1}\right) W_{n_{2}}\left(k_{2}\right) \ldots W_{n_{N}}\left(k_{N}\right)$, with $W_{\mathbf{n}}(\mathbf{k}) \geq 0, \forall \mathbf{k}, \sum_{k_{r},\left|k_{r}\right|<l_{r} r=1, \ldots N} W_{\mathbf{n}}(\mathbf{k})=1$, $\sum_{k_{r},\left|k_{r}\right|<l_{n_{r}} r=1, \ldots N} W_{\mathbf{n}}^{2}(\mathbf{k}) \rightarrow 0$ as $\mathbf{n} \rightarrow \infty$. If $\omega_{\mathbf{j}+\mathbf{k}} \notin[-\pi, \pi]^{N}$, we evaluate $I_{\mathbf{n}, X}\left(\omega_{\mathbf{j}+\mathbf{k}}\right)$ by defining $I_{\mathbf{n}, X}$ to have period $2 \pi$, as in the definition of $f(\omega), \omega \notin[-\pi, \pi]^{N}$.

Theorem 4 Let $\sum_{\mathbf{j} \in \mathbb{Z}^{N}}\left|a_{\mathbf{j}}\right||\mathbf{j}|^{\frac{1}{2}}<\infty$ and $E Z_{\mathbf{1}}^{4}<\infty$. Then

(i) $\lim _{\mathbf{n} \rightarrow \infty} E \widehat{f}(\omega)=f_{X}(\omega)$ and

(ii) $\lim _{\mathbf{n} \rightarrow \infty}\left(\sum_{k_{r},\left|k_{r}\right|<l_{n_{r}}, r=1, \ldots, N} W_{\mathbf{n}}^{2}(\mathbf{k})\right)^{-1} \operatorname{Cov}\left(\widehat{f}(\omega), \widehat{f}\left(\omega^{\prime}\right)\right)$

$$
=\left\{\begin{array}{l}
2 f_{X}^{2}(\omega) \text { if } \omega=\omega^{\prime} \text { and } \omega_{k}=0 \text { or } \pi, \forall k=1, \ldots, N, \\
f_{X}^{2}(\omega) \text { if } \omega=\omega^{\prime} \text { and } 0<\omega_{k}<\pi, \forall k=1, \ldots, N, \\
0 \text { if } \omega \neq \omega^{\prime} .
\end{array}\right.
$$

Proof We have

$$
\begin{aligned}
& \left|E \widehat{f}_{X}(\omega)-f_{X}(\omega)\right| \\
& =\left|\sum_{|k|<l_{n}} W_{\mathbf{n}}(\mathbf{k})\left((2 \pi)^{-N} I_{\mathbf{n}, X}\left(g(\mathbf{n}, \omega)+\omega_{\mathbf{k}}\right)-f_{X}\left(g(\mathbf{n}, \omega)+\omega_{k}\right)+f_{X}\left(g(\mathbf{n}, \omega)+\omega_{\mathbf{k}}\right)-f_{X}(\omega)\right)\right| .
\end{aligned}
$$

Since $l_{n_{r}} \rightarrow \infty, n_{r} \rightarrow \infty$ and $\frac{l_{n_{r}}}{n_{r}} \rightarrow 0$, then

$$
\max _{\left|k_{r}\right| \leq l_{n_{r}}, r=1, \ldots, N}\left\|g(\mathbf{n}, \omega)+\omega_{k}-\omega\right\| \rightarrow 0,
$$

Since $f$ is continuous,

$$
\forall \epsilon>0, \quad \max \left\|f_{X}\left(g(\mathbf{n}, \omega)+\omega_{\mathbf{k}}\right)-f_{X}(\omega)\right\| \leq \frac{\epsilon}{2}
$$

and for $\mathbf{n}$ large enough (see Proposition 10.3.1 in [6]),

$$
\left|(2 \pi)^{-N} I_{\mathbf{n}, X}\left(g(\mathbf{n}, \omega)+\omega_{k}\right)-f_{X}\left(g(\mathbf{n}, \omega)+\omega_{k}\right)\right| \leq \frac{\epsilon}{2},
$$

Since $\sum_{\substack{\left|k_{r}\right|<l_{n r} \\ r=1, \ldots N}} W_{\mathbf{n}}(\mathbf{k})=1$, we have

$$
\left|E \widehat{f}_{X}(\omega)-f_{X}(\omega)\right| \leq \sum_{\substack{k_{r},\left|k_{r}\right|<l_{n_{r}} \\ r=1, \ldots N}} W_{\mathbf{n}}(\mathbf{k}) \epsilon=\epsilon
$$

hence the result. 
(ii) We have

$$
\begin{aligned}
& \operatorname{Cov}\left(\widehat{f_{X}}(\omega), \widehat{f}_{X}\left(\omega^{\prime}\right)\right)=(2 \pi)^{-2 N} \sum_{\substack{\left|j_{r}\right|<l_{n_{r}} \\
r=1, \ldots N}} \sum_{\substack{\left|k_{r}\right|<l_{n_{r}} \\
r=1, \ldots N}} W_{\mathbf{n}}(\mathbf{j}) W_{\mathbf{n}}(\mathbf{k}) \operatorname{Cov}\left(\left(I_{\mathbf{n}, X}\left(g(\mathbf{n}, \omega)+\omega_{\mathbf{j}}\right),\right.\right. \\
& \left.I_{\mathbf{n}, X}\left(g\left(\mathbf{n}, \omega^{\prime}\right)+\omega_{\mathbf{k}}\right)\right) .
\end{aligned}
$$

- If $\omega^{\prime} \neq \omega$, by Theorem 3 ,

$$
\begin{aligned}
\left|\operatorname{Cov}\left(\widehat{f}_{X}(\omega), \widehat{f}_{X}\left(\omega^{\prime}\right)\right)\right| & =\left|(2 \pi)^{N} \sum_{\substack{\left|j_{r}\right|<l_{n_{r}} \\
r=1, \ldots N}} \sum_{\substack{\left|k_{r}\right|<l_{n_{r}} \\
r=1, \ldots N}} W_{\mathbf{n}}(\mathbf{j}) W_{\mathbf{n}}(\mathbf{k}) O\left(\widehat{\mathbf{n}}^{-\frac{1}{2}}\right)\right| \\
& \leq C \widehat{\mathbf{n}}^{-\frac{1}{2}}\left(\sum_{\substack{\left|k_{r}\right|<l_{n_{r}} \\
r=1, \ldots N}} W_{\mathbf{n}}(\mathbf{k})\right)^{2} \\
& \leq C \widehat{\mathbf{n}}^{-\frac{1}{2}}\left(\sum_{\substack{\left|k_{r}\right|<l_{n_{r}} \\
r=1, \ldots N}} W_{\mathbf{n}}^{2}(\mathbf{k})\right)(2 \widehat{\mathbf{k}}+1) .
\end{aligned}
$$

Since $\frac{l_{n_{r}}}{n_{r}} \rightarrow 0$; hence the result.

- In the case $\left.\omega^{\prime}=\omega \in\right] 0, \pi\left[^{N}\right.$,

$$
\begin{aligned}
\operatorname{Var}\left(\widehat{f_{X}}(\omega)\right)= & (2 \pi)^{-2 N} \sum_{\substack{\left|j_{r}\right|<l_{n_{r}} \\
r=1, \ldots N}} W_{\mathbf{n}}^{2}(\mathbf{j}) \operatorname{Var}\left(I_{\mathbf{n}, X}\left(g(n, \omega)+\omega_{j}\right)\right. \\
= & (2 \pi)^{-2 N} \sum_{\substack{\left|j_{r}\right|<l_{n_{r}} \\
r=1, \ldots N}} W_{\mathbf{n}}^{2}(\mathbf{j})\left((2 \pi)^{2} f^{2}\left(g(n, \omega)+\omega_{j}\right)+O\left(\widehat{n}^{-1} \frac{-1}{2}\right)\right) \\
& +(2 \pi)^{-2 N} \sum_{\substack{\left|j_{r}\right|<l_{n_{r}} \\
r=1, \ldots N}} \sum_{\substack{\left|k_{r}\right|<l_{n_{r}} \\
r=1, \ldots N \\
\mathbf{j} \neq \mathbf{k}}} W_{\mathbf{n}}(\mathbf{j}) W_{\mathbf{n}}(\mathbf{k}) O\left(\widehat{\mathbf{n}}^{-1}\right) .
\end{aligned}
$$

Same as (ii), the first term is equal to

$$
\left(\sum_{\substack{\left|j_{r}\right|<l_{n_{r}} \\ r=1, \ldots N}} W_{\mathbf{n}}^{2}(\mathbf{j})\right) f_{X}^{2}(w)+O\left(\sum_{\substack{\left|j_{r}\right|<l_{n_{r}} \\ r=1, \ldots N}} W_{\mathbf{n}}^{2}(\mathbf{j})\right) .
$$

The second term is bounded by

$$
C \widehat{\mathbf{n}}^{-\frac{1}{2}}(2 \pi)^{-2 N}\left(\sum_{\substack{\left|k_{r}\right|<l_{n r} \\ r=1, \ldots N}} W_{\mathbf{n}}^{2}(\mathbf{j})\right)(2 \widehat{\mathbf{k}}+1) .
$$

The rest of the proof is similar to Theorem 10.4.1 in [6]. 

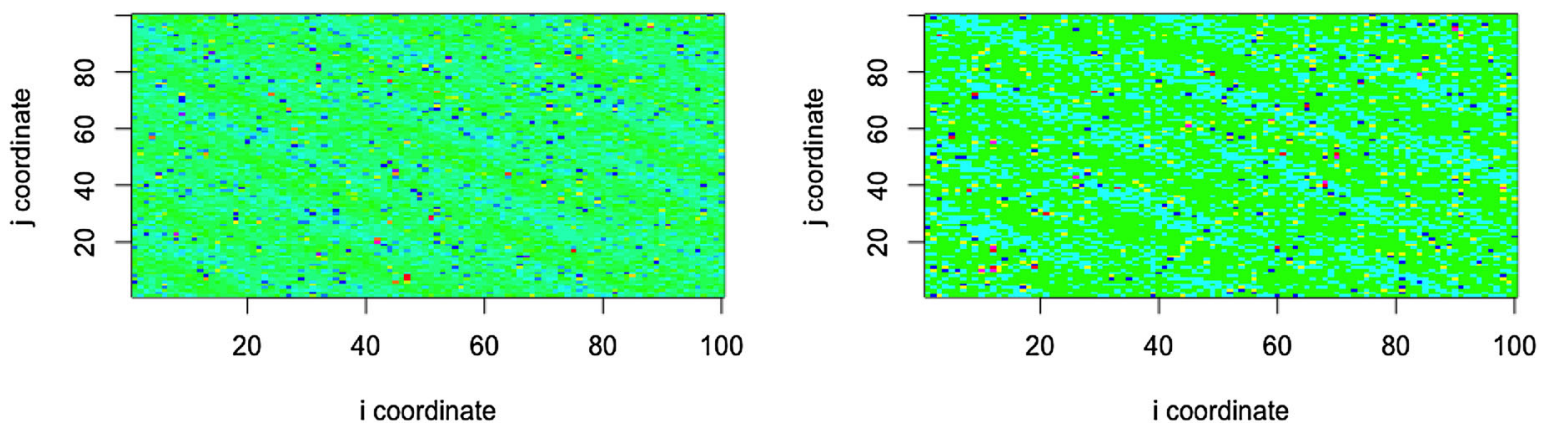

Fig. 1 Image on the right corresponds to $n_{1}=100$ and $\theta=0.5$ while on the left we have $n_{1}=100$ and $\theta=0.9$

\section{Numerical experiments}

In this section, we propose some numerical results of the proposed periodogram towards some simulations. We consider a two-dimensional space $(N=2)$ with the process $\left\{X_{i, j},(i, j) \in \mathbb{Z}^{2}\right\}$ simulated on a rectangular domain $I=\left\{(i, j), 1 \leq i \leq n_{1}, 1 \leq j \leq n_{2}\right\}$ of $n_{1} \times n_{2}$ sites, we take without loss of generality $n_{1}=n_{2}$ for different values of $n_{1} \in\{30,50,100,120\}$. The process is defined by

- $X_{i, j}=0.2 W_{i, j}+\left(Z_{i, j}-\theta Z_{i, j-1}\right)$

- $Z_{i, j}=V_{i, j} V_{i, j-1}$, where the $V i, j$ are independent $\mathcal{N}(0,1)$ distributed random variables

- $W_{i, j}=W_{i, j}^{1}+1.5 W_{i, j}^{2}+2 W_{i, j}^{3}$

- $W_{i, j}^{1}=\sin \left(2 \pi f_{1}(i \cos (\pi / 6)+j \sin (\pi / 6))+n_{1} U_{i, j}^{1}\right.$

$-W_{i, j}^{2}=\sin \left(2 \pi f_{2}(i \cos (\pi / 4)+j \sin (\pi / 4))+n_{1} U_{i, j}^{2}\right.$

- $W_{i, j}^{3}=\sin \left(2 \pi f_{2}(i \cos (\pi / 3)+j \sin (\pi / 3))+n_{1} U_{i, j}^{3}\right.$, with frequencies $f_{1}=2, f_{2}=0.08$ and $f_{3}=0.05$,

$U_{i, j}^{k}, k=1, . ., 3$, and are independently uniformly distributed on $[0,1]$.

All of the following numerical analyses were carried out using the R software (version 3.5.1). Examples of simulated spatial process are given in Fig. 1 for $n_{1}=100$ and $\theta=0.5,0.9$. This figure shows a higher spatial correlation for the process with higher parameter $\theta=0.9$.

To investigate the finite sample properties of the periodogram and a smooth version, we take different values of the parameter $\theta \in\{0.2,0.5,0.9\}$ and two different sample sizes. Taking 300 spatial points $s=\left(s_{1}, s_{2}\right) \in$ $[0,1]^{2}$, we compute the periodogram and a smooth version at 300 frequencies at points $2 s \pi$ including $f_{i}$, $i=1, \ldots, 3$. The results are given in Fig. 2. It clearly shows the higher frequency estimates of $f_{1}=2$, $f_{2}=0.08$ and $f_{3}=0.05$ defined in the model, particularly for large sample sizes, $n_{1}^{2}=10^{4} a n d n_{1}^{2}=144 * 10^{2}$. Note that in order to have the smooth spectral density, we use a weight function obtained by two successive applications of a Kolmogorov-Zurbenko filtering (package ' $k z f s$ ', see [14] for more details).

To assess the performance of the smooth periodogram estimation, particularly at the frequencies $f_{i}, i=$ $1, \ldots, 3$, we consider 100 replications of the simulated model and provide mean estimates. More precisely, we compute $\overline{\hat{f}}_{j}=\frac{1}{100} \sum_{q=1}^{100} \hat{f}_{j}^{q}$, where the $\hat{f}_{j}^{q}$ are the high frequency estimates of $f_{1}=2, f_{2}=0.08$ and $f_{3}=0.05$, from the smooth periodogram computed with the $q-$ th replicated sample. The obtained results are summarized in Table 1 that shows the results of Fig. 2, the good performance of the smooth periodogram estimation for large sample sizes. 

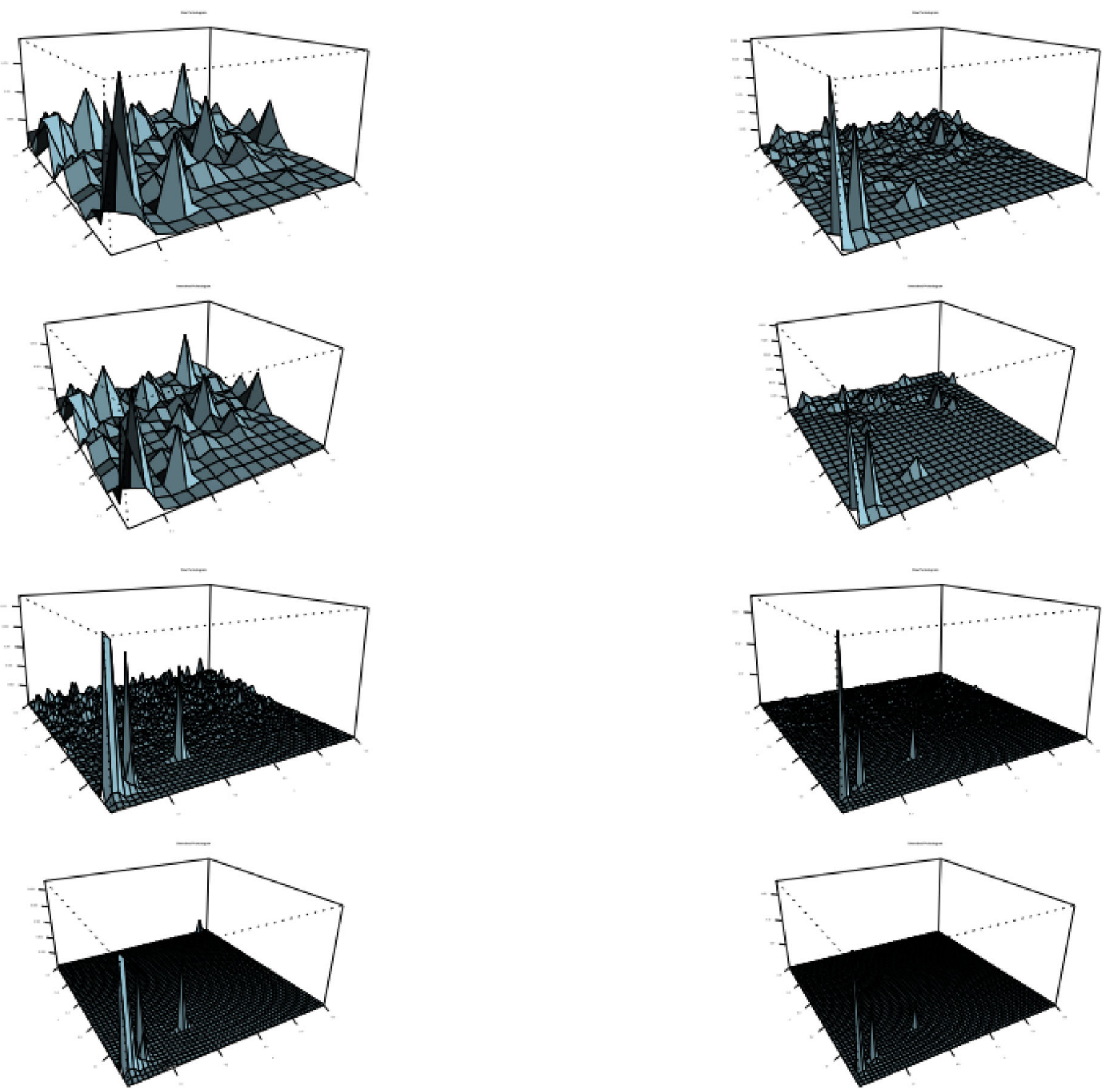

Fig. 2 Image on the top from left to right corresponds to $n_{1}=30$ and $n_{1}=50$ while on the bottom we have from left to right $n_{1}=100$ and $n_{1}=120$; in all situations $\theta=0.9$

Table 1 Means of the frequency estimates of $\hat{f}_{1}, \hat{f}_{2}$ and $\hat{f}_{3}$, from the smooth periodogram, over 100 replications

\begin{tabular}{llll}
\hline Sample size, $\theta$ & $f_{1}=0.2$ & $f_{2}=0.08$ & $f_{3}=0.05$ \\
\hline$n_{1}=n_{2}=100, \theta=0.2$ & 0.1961 & 0.0818 & 0.0511 \\
$n_{1}=n_{2}=100, \theta=0.5$ & 0.1961 & 0.0836 & 0.0497 \\
$n_{1}=n_{2}=100, \theta=0.9$ & 0.1961 & 0.0843 & 0.0491 \\
$n_{1}=n_{2}=120, \theta=0.2$ & 0.2015 & 0.0825 & 0.0486 \\
$n_{1}=n_{2}=120, \theta=0.5$ & 0.2016 & 0.0825 & 0.0486 \\
$n_{1}=n_{2}=120, \theta=0.9$ & 0.2016 & 0.0825 & 0.0486 \\
\hline
\end{tabular}

\section{Conclusion}

In this paper, we have focused on the asymptotic properties of the periodogram of a linear spatial process with $\alpha$ mixing innovations. A relation between periodogram of innovations and the periodogram of the linear 
process is established in spatial case. As the estimator of the spectral density is inconsistent, a linear filter was introduced and the convergence of the smoothed estimate was studied. These studies have shown that the obtained results are similar to those obtained by $[5,6]$ but in a spatial context.

Open Access This article is distributed under the terms of the Creative Commons Attribution 4.0 International License (http:// creativecommons.org/licenses/by/4.0/), which permits unrestricted use, distribution, and reproduction in any medium, provided you give appropriate credit to the original author(s) and the source, provide a link to the Creative Commons license, and indicate if changes were made.

\section{References}

1. Abramenko, V.; Yurchyshyn, V.; Wang, H.; Goode, P.R.: Magnetic power spectra derived from ground and space measurement of the solar magnetic fields. Solar Phys. 201(2), 225-240 (2001)

2. Anselin, L.: Spatial Econometrics: Methods and Models. Kluwer Academic Publishers, Dordrecht (1988)

3. Anselin, L.: Under the hood: issues in the specification and interpretation of spatial regression models. Agric. Econ. 17(3), 247-67 (2002)

4. Barry, R.G.; Perry, A.H.: Synoptic Climatology: Methods and Applications. Methuen \& Co., London (1973)

5. Benrabah, O.; Bensaid, N.: Spectral density estimation for linear process with dependent innovations. Stat. Prob. Lett. 78(12), 1601-1611 (2008)

6. Brockwell, P.J.; Davis, R.A.: Time Series: Theory and Methods, 2nd edn. Springer, New York (1991)

7. Carbon, M.; Tran, L.T.; Wu, B.: Kernel density estimation for random fields. Stat. Probab. Lett. 36, 115-125 (1997)

8. Cressie, N.A.C.: Statistics for Spatial Data. Wiley, New York (1991)

9. Crujeiras, R.M.; Fernandez, R.: On the estimation of the spectral density for continuous spatial process. Statistics 00, 1-14 (2009)

10. Gao, J.; Lu, Z.; Tjøstheim, D.: Moment inequalities for spatial processes. Stat. Probab. Lett. 78, 687-697 (2008)

11. Gelfand, A.E.; Diggle, P.J.; Fuentes, M.; Guttorp, P. (eds.): Handbook of Spatial Statistics. Chapman Hall/CRC Press, Boca Raton (2010)

12. Guo, D.; Mennis, J.: Spatial data mining and geographic knowledge discovery: an introduction. Comput. Environ. Urban Syst. 33, 403408 (2009)

13. Haining, R.: Spatial Data Analysis Theory and Practice. Cambridge University Press, Cambridge (2003)

14. Luo, M.; Zurbenko, I.: Multi-Scale Motions Separation with Kolmogorov-Zurbenko Periodogram Signals. 2016.9.23. R package kzfs. https://CRAN.R-project.org/package $=$ kzfs

15. Miller, H.J.: Toblers first law and spatial analysis. Ann. Assoc. Am. Geograph. 94, 284289 (2004)

16. Smith, M.J.: Statistical Analysis Handbook . www.Statsref.com (2015). Accessed 21 Dec 2017

17. Stein, M.L.: Fixed domain asymptotic for spatial periodogramms. J. Am. Stat. Assoc. 90, 1277-1288 (1995)

18. Tran, L.T.: Kernel density estimation on random fields. J. Multivar. Anal. 34, 37-53 (1990)

19. Vidal-Sanz, J.M.: Automatic spectral density estimation for random fields on a lattice via bootstrap. Test Off. J. Span. Soc. Stat. Oper. Res. 18(1), 96-114 (2009). Springer

Publisher's Note Springer Nature remains neutral with regard to jurisdictional claims in published maps and institutional affiliations. 